

\section{REALIZING ARMENIA'S HIGH-TECH POTENTIAL}

January 2020

ॠ WORLD BANK GROUP 
(c) 2020 The World Bank

1818 H Street NW, Washington DC 20433

Telephone: 202-473-1000; Internet: www.worldbank.org

Some rights reserved

This work is a product of the staff of The World Bank. The findings, interpretations, and conclusions expressed in this work do not necessarily reflect the views of the Executive Directors of The World Bank or the governments they represent. The World Bank does not guarantee the accuracy of the data included in this work. The boundaries, colors, denominations, and other information shown on any map in this work do not imply any judgment on the part of The World Bank concerning the legal status of any territory or the endorsement or acceptance of such boundaries.

\section{Rights and Permissions}

The material in this work is subject to copyright. Because The World Bank encourages dissemination of its knowledge, this work may be reproduced, in whole or in part, for noncommercial purposes as long as full attribution to this work is given.

Attribution-Please cite the work as follows: "World Bank. 2020. Realizing Armenia's Technology Potential. Washington, D.C.: World Bank.”

All queries on rights and licenses, including subsidiary rights, should be addressed to World Bank Publications, The World Bank Group, $1818 \mathrm{H}$ Street NW, Washington, DC 20433, USA; fax: 202-522-2625; e-mail: pubrights@worldbank.org. 


\section{CONTENTS}

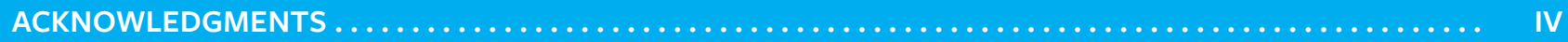

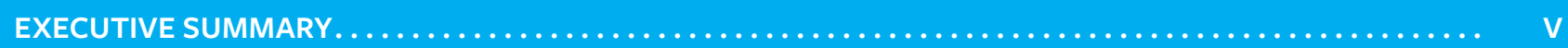

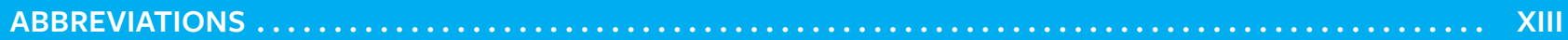

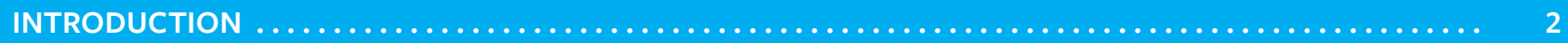

CHAPTER 1

ARMENIA'S CURRENT POSITIONING IN HIGH-TECH GVCS $\ldots \ldots \ldots \ldots \ldots \ldots \ldots \ldots \ldots \ldots \ldots \ldots \ldots \ldots \ldots \ldots \ldots$

\section{CHAPTER 2}

STRATEGIC GVC REPOSITIONING OPTIONS FOR ARMENIA $\ldots \ldots \ldots \ldots \ldots \ldots \ldots \ldots \ldots \ldots \ldots \ldots \ldots \ldots \ldots$

2.1 Leveraging Armenia's factor conditions to develop global niche activities 9

2.2 Upgrading to “adjacent” higher-value-added activities 12

2.3 Building capacity and relevance in nascent technologies while they are still accessible 19

\section{CHAPTER 3}

STRENGTHENING THE ECOSYSTEM. .

\section{CHAPTER 4}

ACTIONS AND RECOMMENDATIONS

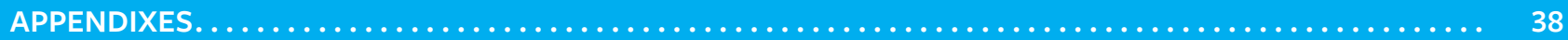

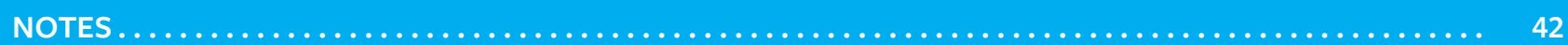

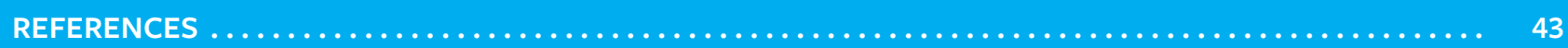

\section{Figures}

1.1 ICT services exports vs. other high-tech exports, 2009-17

1.2 ICT services exports vs. total goods and services exports, 2009-17 5

1.3 ICT services exports per capita, Armenia and regional high performer comparators, 1995-2017 6

$1.4 \quad$ ICT exports per capita vs. CAGR of ICT services exports

1.5 Turnover of Armenian ICT firms by type 8

2.1 Market size through 2023 for 15 clean technologies in emerging economies

2.2 Size of the clean technology market accessible to SMEs in Eastern Europe

2.6 NSMS vs. ICT

2.7 Monthly average salaries of faculty in public universities in 28 countries, 2012

2.8 Capital expenditure requirements, firm consolidation, and barriers to entry are different in different segments 18

2.9 Citations of Armenian research papers in international journals, 2018

CS2.1 Male cancer mortality rates in 183 countries, 2016

CS2.2 Structure of the digital health GVC (at intersection with biotech and genomics) 26

CS2.3 Armenia's participation in the biotech/digital health value chain 27

\section{Tables}

ES.1 Actions and recommendations ix

CS2.1 Health care expenditure per capita, 2016

4.1 Summary of recommendations 34 


\section{ACKNOWLEDGMENTS}

This report was written by Ifeyinwa Onugha under the guidance of Wolfgang Fengler. Special thanks to Gohar Malumyan for her data analysis and her insights and contributions relating to local trends. Thanks also for contributions and insights from World Bank Group colleagues and associates, including Wim Douw, Tafadzwa
Dube, Alejandro Espinosa-Wang, Lillyana Daza Jaller, Martin Molinuevo, Arsen Nazaryan, Alanna Simpson, and Siegfried Zottel. Thanks also to firms and individuals in Yerevan, Silicon Valley, and Washington D.C. who were willing to participate in consultations to aid the preparation of this report. 


\section{EXECUTIVE SUMMARY}

\section{Service exports remain Armenia's best strategy to achieve higher exports and create well-paid jobs.}

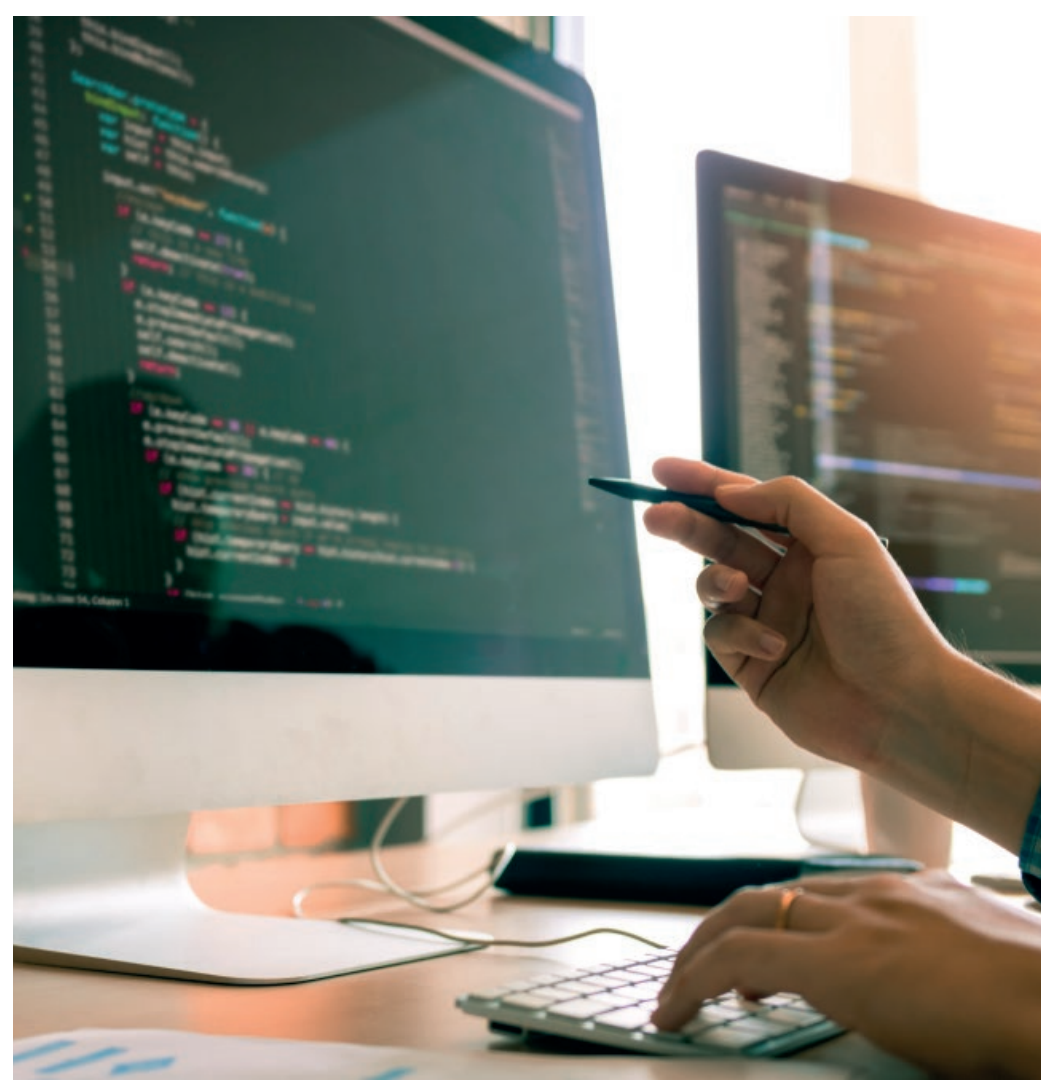

Armenia's economy is heavily reliant on domestic demand, which is itself driven by remittances that support local construction and consumption. Armenia will need to shift to an exportled strategy if it is to move from middleincome to high-income status. Because Armenia is a landlocked country with comparatively high trading costs in physical goods, high-tech digital exports will continue to be key to Armenia's growth. This report seeks to identify strategic opportunities for Armenia's private sector in high-tech global value chains (GVCs) and also highlights reforms that could support Armenia's evolution. The study builds on the FY18 Armenia Systematic Country Diagnostic, which identifies value-chain development as a core building block for sectoral growth. Although the report is targeted at policy makers in the Armenian government, sectoral development strategies rely heavily on strong private sector engagement and open public-private dialogue. Armenia's tech community may therefore also take interest in this analysis. 


\section{ARMENIA'S CURRENT POSITIONING IN HIGH-TECH GLOBAL VALUE CHAINS}

Armenia's participation in information and communication technology (ICT) services GVCs has resulted in promising export gains. ICT services exports doubled between 2009 and 2017, growing from about US\$94 million to US\$212 million. Most of this export activity is in software programming, building on a strong history of outsourcing services. Armenian ICT firms are moving away from a service model and toward product design. Several firms (e.g., Synopsys and Mentor Graphics) are already integrated into GVCs through foreign subsidiaries, and a vibrant high-tech start-up movement has produced high-profile firms that have achieved global success. As an example, PicsArt, valued at US\$250 million, has developed an image-editing and social-networking app that has been downloaded 600 million times. Another mobile app developer with its origins and development team based in Armenia, SoloLearn, has 1 million active monthly users globally.

While export growth is encouraging, Armenian firms may struggle to stay competitive in high-tech GVCs if they continue to target highly saturated, mass-market vertical sectors. Armenian firms are competing globally to deliver mass-market software and hardware solutions for a range of vertical applications. Examples include human resource management software development, lifestyle mobile applications, banking software, social media software, and educational training kits. Many of these solutions are disconnected from other high-tech activities in the value chain, limiting opportunities for upgrading or vertical integration. In addition, because the customer base for many of these applications is often far from Armenia, generating new business has proven difficult. Remaining competitive may be a challenge for Armenia as global competition from artificial intelligence (AI)-enabled technologies (and lower-wage countries) increases.

As high-tech GVCs evolve, the Armenian government can strategically support the country's migration toward activities that are catalyzedrather than threatened-by emerging technologies. Armenia's tech community is poised to move beyond software development toward deeper participation in the development and commercialization of emerging and disruptive technologies. The government can facilitate this transition by strategically repositioning Armenia in (a) activities that leverage Armenia's factor conditions, (b) 'adjacent' higher-value-added activities that build on the existing competencies of Armenian firms, and (c) the development of nascent technologies while they are still accessible. In addition, there are a number of areas where the regulatory ecosystem could be strengthened. These opportunities are discussed below. A caveat, however: the sectoral examples highlighted in the following sections are relevant at the time of writing. As the tech industry rapidly evolves, some of the presented examples may “expire," giving way to new opportunities in other emerging technologies.

\section{STRATEGIC GVC REPOSITIONING OPPORTUNITIES FOR ARMENIA}

\section{LEVERAGING ARMENIA'S FACTOR CONDITIONS TO DEVELOP GLOBAL NICHE SECTORS}

Armenian firms can target global niche vertical applications that leverage Armenia's comparative advantages and, in so doing, attract foreign firms to Armenia, thus "localizing" the global market. Armenia has several special characteristics (e.g., data, geology, population characteristics, geography, and industrial history) that make it particularly conducive to developing solutions for certain vertical applications. With the support of strategic policies and investments, Armenian firms can build competence in these niche higher-value-added activities. In addition, the government can build on these factor conditions by implementing a targeted investment promotion strategy to attract global firms in these niche sectors to Armenia. In so doing, the government could "bring the global market to Armenia," reducing information asymmetries and encouraging close cooperation and collaboration between global firms and local innovators and start-ups.

Biotech solutions, cleantech and seismic engineering are examples of vertical applications with rich potential for Armenia's software and hardware development community. Armenia's population has distinctive health characteristics that could be valuable to global biotech development. As the government codifies these health characteristics through its digital health record system, Armenia's software development community can build on these assets as global demand for digital health care solutions widen to include a 
variety of users such as physicians, insurance companies, research centers and pharmacies. With an appropriate regulatory framework, Armenia can position itself as a global hub for digital health, biotech development and bioscience research. As a second example, Armenia's mining history has resulted in significant ongoing air and water pollution and land contamination. The "cleantech" industry could mitigate some of this environmental damage. Not only does the global cleantech industry have very strong growth potential, small and medium enterprises in middle- and low-income countries will have important roles to play over the next decade. Rather than relying on the local mining industry as a market, Armenia can become a concentrated research, development, and prototyping testbed for global cleantech firms. Finally, Armenia's geology could also lend itself to geographical information system development, seismic engineering, and disaster risk management technologies.

\section{UPGRADING TO “ADJACENT” HIGHER-VALUE- ADDED ACTIVITIES}

GVC upgrading can come through vertical integration of value-chain activities that are adjacent to Armenia's existing ICT services exports. Policy makers can examine the high-tech activities that Armenian firms already perform well and implement targeted programs to support vertical integration into “adjacent" higher-value-added activities. This report examines opportunities for upgrading into (a) AI research and engineering (building on Armenia's competence in mathematics and computer science) and (b) semiconductor design (building on Armenia's strong foothold in electronic design automation). ${ }^{1}$

\section{》AI RESEARCH AND ENGINEERING}

With investment in tertiary research, Armenia's strong heritage in mathematics could position it as a global hub for pure AI research. Pure AI research (the creation of brand-new algorithms) relies on advanced mathematical techniques. Armenia has a strong global comparative advantage in mathematics and natural sciences-even more so than in ICT. Until recently, commercial opportunities for mathematicians in Armenia have been few, but AI research has created high demand for mathematicians globally. Armenia already has some nascent activity in AI, but a lack of tertiary training is leading to a brain drain and undermining local investment opportunities. The government can build an AI PhD program in Armenia and identify opportunities for joint research labs in collaboration with leading international research institutions (in both the public and the private sector) in this field.

With a stronger AI research base, the government can implement policies to facilitate access to data and to collect and share both administrative and statistical data for AI engineering. Success in AI engineering (adapting and applying existing AI algorithms to "real-world" applications) will be contingent on access to proprietary data. Gaining access to foreign private sector data will require Armenian firms to comply with global information security and management provisions, such as the ISO/IEC ${ }^{2}$ 27000-series and the European Union General Data Protection Regulation 2016/679. The government can implement training programs for private firms to build compliance and meet international standards. The government can go further to help plug the gap in available data by improving the accessibility of its own data. Where regular data collection is already taking place, policies and practices differ significantly between ministries, and there is no clear policy or necessary infrastructure governing efficient data exchange in the government-to-government, government-to-business, and government-to-consumer domains. The government can consider standardizing the collection of both administrative and statistical data in addition to designing policies relating to the classification, formatting, storage, and exchange of these data.

\section{»SEMICONDUCTOR DESIGN}

Upgrading to fabless semiconductor design and Intellectual Property development is an attractive prospect, but extremely high start-up costs, distance to market and Wassenaar Agreement export controls are holding Armenia back. Synopsys, a global leader in electronic design automation (EDA), is Armenia's largest ICT employer, with 800 employees. Considering Synopsys together with Mentor Graphics, Armenia has a core capability in EDA software for semiconductor design. In cities with vibrant semiconductor ecosystems, relatively small start-up firms are able to spin off from larger firms to create their own "fabless" semiconductor firms ${ }^{3}$ or design "intellectual property (IP) cores"-the building blocks of semiconductors. The value-added associated with these activities is extremely high, but start-up costs are far higher than the venture capital currently available in Armenia. Although Cisco Systems (through acquisition 
of U.S. firm Memoir Systems) has begun IP core and fabless semiconductor design, this important milestone may be difficult to replicate without heavy foreign investment capital or an aggressive ramp-up in venture capital funding in Armenia. In addition, Wassenaar Agreement export controls limit the extent to which international firms can collaborate with Armenian firms in semiconductor design due to Armenia's categorization as a Group D country by the U.S. Department of Commerce. Strong lobbying and intervention by the Armenian government will be needed to re-assign in the grouping or seek blanket exemptions.

\section{BUILD CAPACITY AND RELEVANCE IN NASCENT TECHNOLOGIES WHILE THEY ARE STILL ACCESSIBLE}

\section{»QUANTUM COMPUTING}

As emerging technologies continue to disrupt GVC structures, quantum computing presents an opportunity for Armenian firms to actively participate in evolving GVCs, rather than lagging behind.

Quantum computing research is still in its infancy. It is true that only a handful of firms globally have the capital, knowledge, and convening power to construct prototype quantum computers, outside of hardware development the quantum research ecosystem is vast, and the global playing field is level. In addition, private sector funding (rather than state-funded programs) now dominates quantum research, opening the door to researchers in middle- and low-income countries.

Global citations of Armenia's research and development (R\&D) in physics far exceed that of computer sciences and even mathematics, placing Armenia in the top 25 countries in the world for R\&D citations in nuclear physics. However, Armenia has no national quantum strategy and is not leveraging its potential comparative advantage in this area.

As the global race intensifies, quantum computing strategy and road map will be increasingly important as will the need to invest in tertiary education and research. The Armenian government could consider policies to encourage international collaboration in the field of quantum research (e.g., applied research grants, competitive research collaborations, competitions, and R\&D tax credits) and position Armenia as a regular "stop" on the global quantum computing conference circuit. This should include formal tertiary training at all three levelsbachelor's, master's, and doctoral.

\section{STRENGTHENING THE ECOSYSTEM}

\section{Innovation and entrepreneurship are constrained} by limited investment capital; in addition, corporate law is poorly suited to early stage financing and investment. There is only limited venture capital available in Armenia to support business innovation and entrepreneurial endeavors. At the same time, FDI opportunities are constrained by a 1994 law that does not adequately address current investment realities. As an example, the law does not allow investors to choose international courts over local courts in case of arbitration. In addition, some aspects of Armenia's corporate law are poorly suited to early stage financing and investment. As a result of these bottlenecks, start-ups in Armenia's tech community typically incorporate abroad to attract investment, limiting local tax revenue and spillover effects.

Growth financing gaps could be bridged by introducing innovative FinTech products and providing targeted financial capability advisory services to micro, small, and medium enterprises (MSMEs). Small firms in Armenia rely heavily on internal resources and retained earnings for growth finance. The limited use of bank financing is related to the high cost of borrowing in Armenia. Promotion of FinTech innovations (e.g., mobile banking) and use of agents could help to expand outreach to underserved segments and locations. Expanding targeted financial capability advisory services to MSMEs on financial products, financial reporting, financial management, business planning and marketing would enhance their bankability and discourage informality, which is already at a very high level in the enterprise sector.

Armenia already has a fairly strong framework in the area of electronic transactions and data governance, but these laws could be strengthened and complemented by a framework to address intermediary liability. The government could consider expanding the Law on Electronic Document and Electronic Signature to recognize all electronic signatures and could, in addition, grant them "presumption of validity and authenticity" in legal proceedings. Armenia already has comprehensive legislation to protect online personal data privacy. However, the Agency for the Protection of Personal Data could consider including specific regulations for cybersecurity, adding requirements such as the appointment of a data manager and risk assessment 
procedures. Finally, the government could consider a framework that explicitly addresses intermediary liability, with safe harbor provisions to shield firms from liability for infringing content posted by third parties, including intellectual property violations.

Armenia's potential in high-tech sectors is closely tied with its R\&D development landscape and tertiary education framework; both are in need of targeted reforms. An increase of public funding for R\&D could support targeted research policies, including applied research grants, competitive research collaborations, competitions, and R\&D tax credits. At the same, tertiary courses in engineering and computer science are in urgent need of overhaul. Additionally, universities can better work with the private sector to provide more professional development and on-the-job training options that open up mid-career learning options.

\section{NOTES}

1. Electronic design automation software comprises applications used by the semiconductor industry to design chips.

2. ISO stands for International Organization for Standardization. IEC stands for International Electrotechnical Commission.

3. "Fabless" semiconductor firms design and sell complete chipsets but outsource their fabrication.

》Develop an innovation policy road map that identifies policies that encourage international research collaboration in priority fields.

\begin{tabular}{|c|c|c|c|}
\hline$\gg$ & $\begin{array}{l}\text { Provide support for research and innovation programs through } \\
\text { sub-financing, e.g., applied research grants, competitive } \\
\text { research collaborations and matching grants programs for } \\
\text { start-ups. }\end{array}$ & $\begin{array}{l}\text { MoESCS, } \\
\text { MoHTI }\end{array}$ & Short term \\
\hline$\gg$ & $\begin{array}{l}\text { Strengthen human resources, research excellence and research } \\
\text { commercialization through government-facilitated collaborative } \\
\text { research programs with international universities, private } \\
\text { sector, and a targeted visa program. }\end{array}$ & MoESCS & Short term \\
\hline$\gg$ & $\begin{array}{l}\text { Develop capacity of technology transfer offices to convert early } \\
\text { stage research to market-ready technology and complete deals. }\end{array}$ & $\begin{array}{l}\text { MoESCS, } \\
\text { MoHTI }\end{array}$ & Long term \\
\hline$\gg$ & $\begin{array}{l}\text { Support development of local and diaspora-led business angel } \\
\text { networks in Armenia. }\end{array}$ & MoHTI, MoE & Long term \\
\hline$\gg$ & $\begin{array}{l}\text { Shift focus of publicly funded seed-funding programs (including } \\
\text { incubators and accelerators) toward "successful exits" rather } \\
\text { than "quantity of firms" in the program. }\end{array}$ & MoHTI & Short term \\
\hline
\end{tabular}


》Introduce software patenting legislation to protect software that demonstrates "further technical effect" (in line with EU guidelines).

》 Introduce managerial training programs to build middlemanagement capacity.

\section{Access to growth finance}

》 Launch consultations and develop a fintech strategy to MoF, CBA Short term introduce innovative products that could help bridge the MSME financing gap.

》Develop implementation strategy (including adequate regulatory safeguards and oversight) to use agents (e.g., the Armenian Post) for delivery of core financial services.

Expand targeted financial capability advisory services to MSMEs on financial products, financial reporting, financial management, business planning and marketing to enhance their bankability and discourage informality.

\section{Corporate law and foreign direct investment}

» Introduce debt instruments (e.g., convertible loans) that can be converted to equity in subsequent funding rounds.

》 Introduce corporate entities that support different classes of voting shares (with differing entitlements to dividends and/or different levels of voting power) in order to better reflect early stage financing risks.

》Develop clear rules pertaining to the fiduciary responsibilities of corporate management teams toward minority shareholders (so that the latter are adequately protected-particularly where the interests of minority shareholders directly clash with those of majority shareholders).

》 Introduce legislation that allows investors to choose between local courts and international courts in the event of arbitration.

》Strengthen investment "aftercare" by creating clear mechanisms to identify, track, and manage grievances between investors and public agencies before they escalate into disputes or withdrawals.

\section{SRLE, MoE, MoF \\ Short term}

SRLE, MOE

Short term

\section{SRLE, MoE Long term}


》 Repeal 1994 Foreign Investment Law and enact new umbrella framework for investment in line with international best practice, including:

> Defining scope of application of the "Fair and Equitable Treatment" principle,

> Defining characteristics of compensation payment,

> Defining limits of prohibition of direct and indirect expropriation, and

> Proscribing the rights of the investor to dispose of their profits and export their funds.

》 Build "investor roadmap" to identify permits, processes and administrative changes that are constraining investments.

Design investment promotion strategy to attract global firms in niche sectors that can leverage Armenia's particular factor conditions (e.g., biotech and cleantech).

\section{Digital trade}

\Expand the Law on Electronic Document and Electronic Signature to recognize all electronic signatures and grant them, in addition, "presumption of validity and authenticity" in legal proceedings.

》 Include specific regulations for cybersecurity in the Law on Personal Data Protection, adding requirements such as the appointment of a data manager and risk assessment procedures.

》Develop framework that explicitly addresses online intermediaries, with safe harbor provisions to shield them from liability for infringing content posted by third parties, including intellectual property violation.

》 Work with the U.S. Department of Commerce and international counterparts to improve Armenia's grouping in order to facilitate trade and collaboration in "dual-use" technologies as per the international Wassenaar Agreement.

\section{Data policy}

» Implement policies relating to the classification, formatting, and storage of administrative and statistical data.
MoJ
Long term

MoJ

Long term

Implement policies that will enhance digital data management capacity and promote digitization among private firms.
MoHTI, MoE Short term

MOHTI, MOE

Short term 
》 Implement localization and data sovereignty laws that do not stifle interest but still stimulate local economic activity in Armenia.

》Provide technical assistance to help the private sector comply with the EU's General Data Protection Regulations and meet international standards (e.g., the ISO/IEC 27000 family of standards).

\section{Education}

》 Integrate planned "High-Tech Industries Strategy" with planned MoESCS, MoHTI

Short term "Education, Science and Culture, and Sports Strategy."

》 Update bachelor's, master's, and PhD curricula in computer science and engineering fields to correspond with industry standards.

》Introduce annual curriculum review process that uses publicprivate dialogue process to solicit industry opinion and incorporates clear mechanism for revisions and updates.

》B Build an AI PhD program and identify opportunities for joint MoESCS, MoHTI Short term research labs in collaboration with leading international research institutions (in both the public and the private sector) in this field.

》Develop a quantum computing strategy and road map. This should include formal tertiary training in quantum computing at all three levels-bachelor's, master's, and doctoral.

》S Strengthen future incarnations of the "Programme for MoF, MoE, MoESCS Short term Anti-Corruption Measures in Education" as follows:

> Provide adequate funding for coordination, monitoring, and implementation of all listed anti-corruption measures,

> Reduce prevalence of short-term, noncompetitive appointments to increase employment security and predictability among university staff,

> Introduce compliance and integrity into accreditation and reaccreditation criteria for higher education providers, and

> Develop comprehensive corruption detection and reporting mechanisms in the higher education sector, in such a way as to protect whistleblowers. 


\section{ABBREVIATIONS}

AGP Armenian Genome Project

AI artificial intelligence

ArMed Armenia National Electronic Health Information System

CBA Central Bank of Armenia

DNA deoxyribonucleic Acid

EDA electronic design automation

EIF Enterprise Incubator Foundation

EHR electronic health records

FDI foreign direct investment

FPGAs field programmable gate arrays

GDP gross domestic product

GIS geographical information system

GVC global value chain

ICT information and communication technology

IEC International Electrotechnical Commission

IoT internet of things

IP intellectual property

ISO International Organization for Standardization

ISTC Innovative solutions and Technologies Center

MIPT National Research University of Electronic Technology

MoE Ministry of Economy

MoESCS Ministry of Education, Science, Culture, and Sports

MoF Ministry of Finance

MoHTI Ministry of High-Tech Sectors

MSME micro, small, and medium enterprise

NPUA National Polytechnic University of Armenia

NSMS Natural Sciences, Mathematics and Statistics

NSSP National Service for Seismic Protection

ACN Anti-Corruption Network for Eastern Europe and Central Asia

R\&D research and development

SME small and medium enterprises

SRLE State Register of Legal Entities, Ministry of Justice of the Republic of Armenia

WA Wassenaar Arrangement 


\section{INTRODUCTION}

Armenia is poised to catalyze growth in high-tech sectors. In the spring of 2018, large, peaceful nationwide street protests (referred to as Armenia's "velvet revolution") resulted in new leadership, which went on to consolidate a majority following parliamentary elections in December 2018. With the new government's commitment to regulatory reform, there is an opportunity to create a much stronger, start-upfriendly business environment that better enables the private sector to increase its productivity, accelerate growth, and attract talent and foreign direct investment into the country. In addition, a new Ministry of High-Tech Industry has been created to design and implement policies and programs that will catalyze growth.

Emerging digital technologies are changing the structure of global value chains (GVCs) in high-tech sectors. With the world now undergoing a fourth industrial revolution (Industry 4.0), Armenia needs to better position itself to leverage global digital trends. The World Development Report 2020: Trading in the Age of Global Value Chains highlights the impact that technological progress has on GVC participation, including reducing trading costs, inducing quality upgrading and churn, and shifting relative productivity across sectors. The data economy can affect the geography of production by reducing the importance of wage costs, changing delivery models, and responding to changing consumer preferences. To the extent that the internet of things (IoT), advanced robotics, $3 \mathrm{D}$ printing, and other digital technologies associated with Industry 4.0 are labor-saving and intensify the "servicification" of manufacturing, they render high-skill (and often high-wage) economies more attractive for manufacturing. In addition, the platform economy matches buyers and suppliers from all over the world in new delivery models to create new markets. Armenia needs to be ready to adapt and leverage these new digital technologies and upskill its workforce or else risk falling behind.

The purposes of this report are (a) to help policy makers identify strategic opportunities for Armenia's private sector in high-tech GVCs as they evolve and (b) to highlight reforms that could support this repositioning. As digital technologies continue to influence the structure of high-tech GVCs and as lower-wage countries continue to increase their market share, Armenia's policy makers and private sector alike must be more strategic about GVC development and upgrading. This report seeks to identify strategic opportunities for Armenia's private sector in high-tech GVCs and also highlights reforms that could support Armenia's evolution. This study builds on the fiscal 2018 Armenia Systematic Country Diagnostic (World Bank 2017), which identifies value-chain development as a core building block for sectoral growth. 


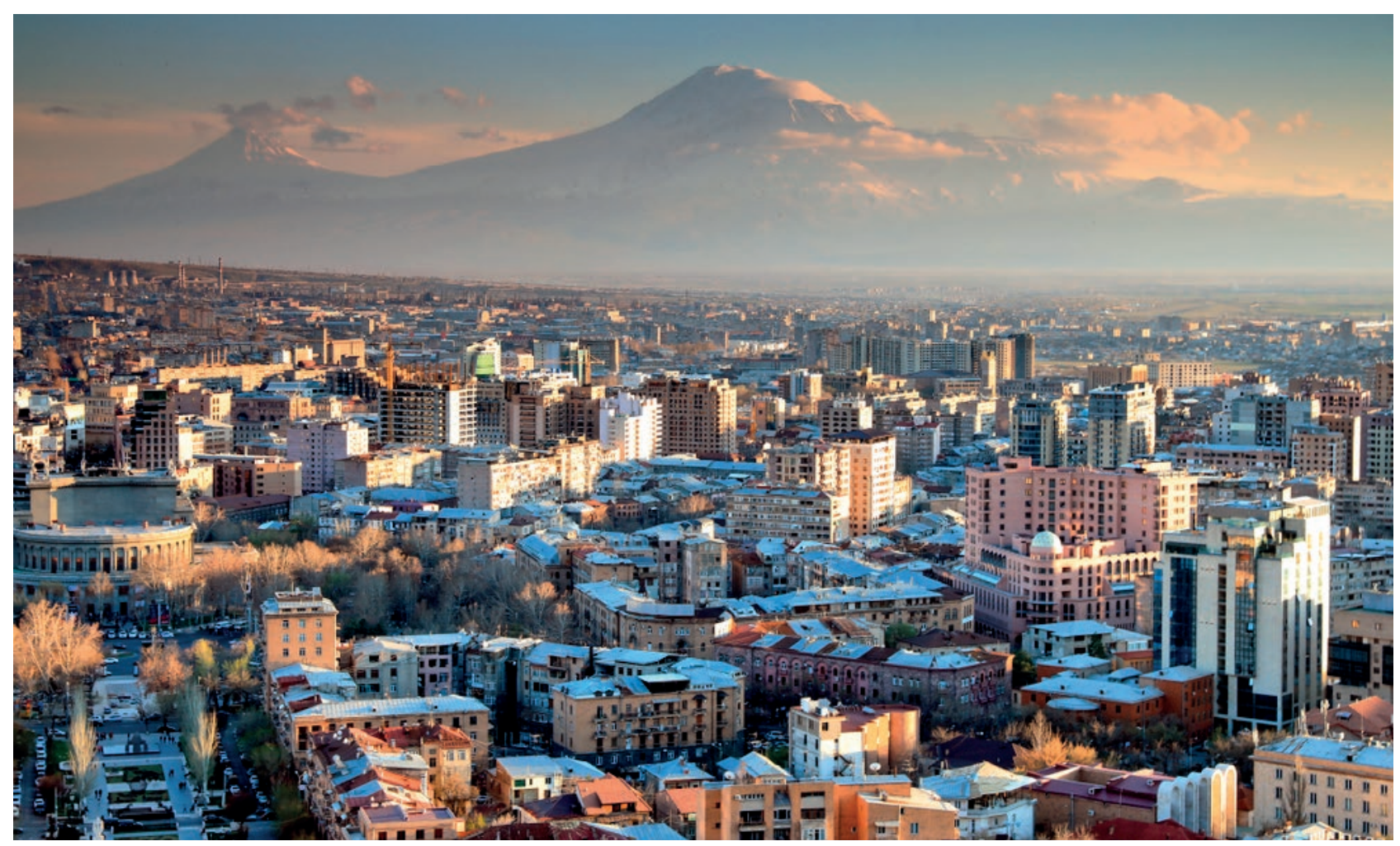

The report uses a qualitative analytical approach $^{1}$ to inform policy design and help the Armenian government identify strategic options to retain a greater share of value added from global industries. The approach uses primary research methods and interviews to identify the strategic position of Armenia's high-tech firms in the GVCs they serve. In particular, the approach appraises the capacity of Armenian firms to: (a) create value in the GVCs in which they participate, (b) guard their competitive positions, and (c) upgrade to higher-value-added activities. Consultations included almost 50 private firms, investors, and learning institutions from Yerevan and from San Diego, Silicon Valley, and Washington D.C. in the U.S. Armenia's performance in identified segments was assessed compared with regional, as well as global, competitors, and the "ideal value chain" for those segments was compared with the Armenian context.

\section{These sectoral development strategies will rely} heavily on strong engagement with Armenia's private sector high-tech community. Successful design and implementation of sector-specific strategies relies heavily on intensive interaction between project teams, private sector beneficiaries and cluster organizations. Any recommendations arising from this report should only be implemented through enhanced stakeholder involvement and facilitated public-private dialogue with Armenia's high-tech community may also take an interest in this analysis.

This report consists of four chapters. Chapter 1 describes Armenia's current position in high-tech GVCs. Chapter 2 focuses on strategic repositioning and upgrading options for Armenia. Chapter 3 describes opportunities to strengthen Armenia's regulatory ecosystem. Lastly, chapter 4 describes recommended actions for strengthening Armenia's participation in high-tech GVCs through policy reforms, capital investments, and skills development. 


\section{ARMENIA'S CURRENT POSITIONING IN HIGH-TECH GVCS}

While information and communication technology

(ICT) services dominate armenia's high-tech export activity, it is still a relatively small share of the overall economy. Armenia's international trade in high-tech sectors is dominated by ICT services, which far outweigh tangible ICT goods exports (see figure 1.1). Exports in ICT services more than doubled between 2009 and 2017, growing from about US\$94 million to US\$212 million. In contrast, other high-tech exports (e.g., aerospace, pharmaceuticals, scientific instruments, and electrical machinery) reached only US\$26 million in 2017, and other ICT goods exports reached only US\$4 million. Although growth in Armenia's ICT services exports is encouraging, these exports comprised only 11 percent of total service exports and only 4.8 percent of goods and services exports in 2017 (see figure 1.2).

Growth in ICT exports compares favorably with regional competitors, but there is still room for improvement. Armenia's export performance compares favorably with some of its regional competitors, including Georgia, Kazakhstan, the Russian Federation, and Ukraine (see figure 1.3). In addition, Armenia outperforms global ICT services giants China and India on a per capita basis (see figure 1.4). However, there is still room for improvement; Belarus, Bulgaria, Hungary, Lithuania, Poland, Romania, Slovenia, and the Slovak Republic exported between
US $\$ 150$ to US $\$ 250$ in ICT services per capita in 2017 , compared with Armenia's US $\$ 72$ in ICT services per capita. (Exports per capita of global leaders are far higher, in excess of US\$1,000 per capita in 2017.)

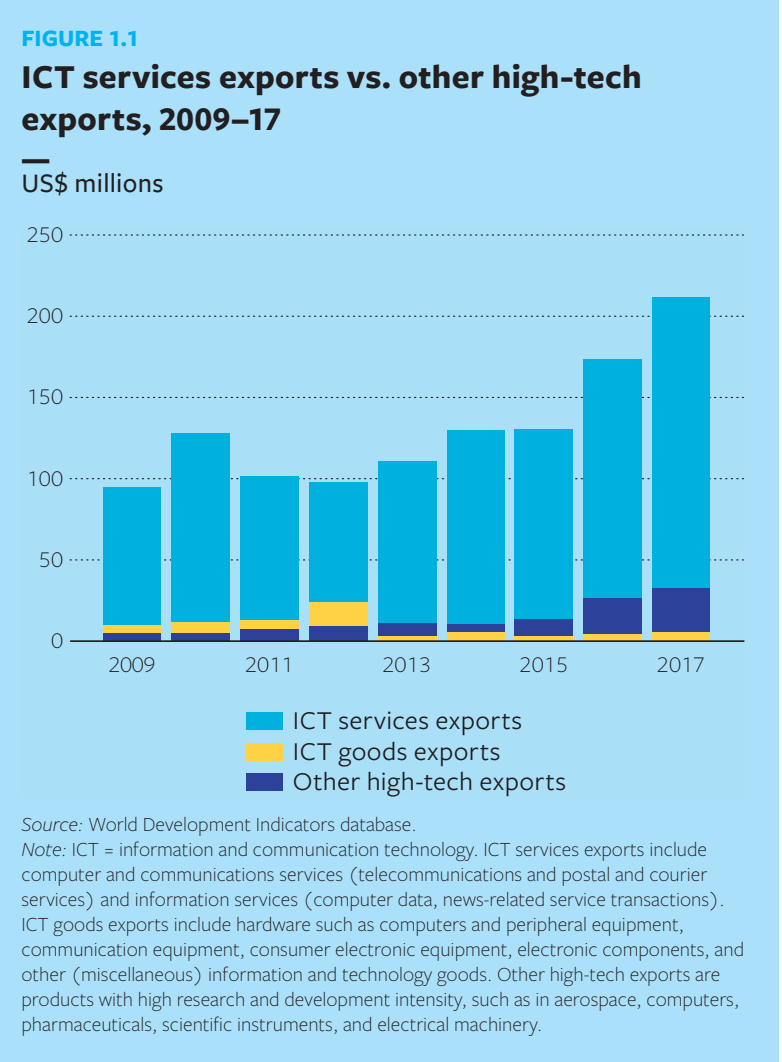

FIGURE 1.1

ICT services exports vs. other high-tech exports, 2009-17

US\$ millions

250 


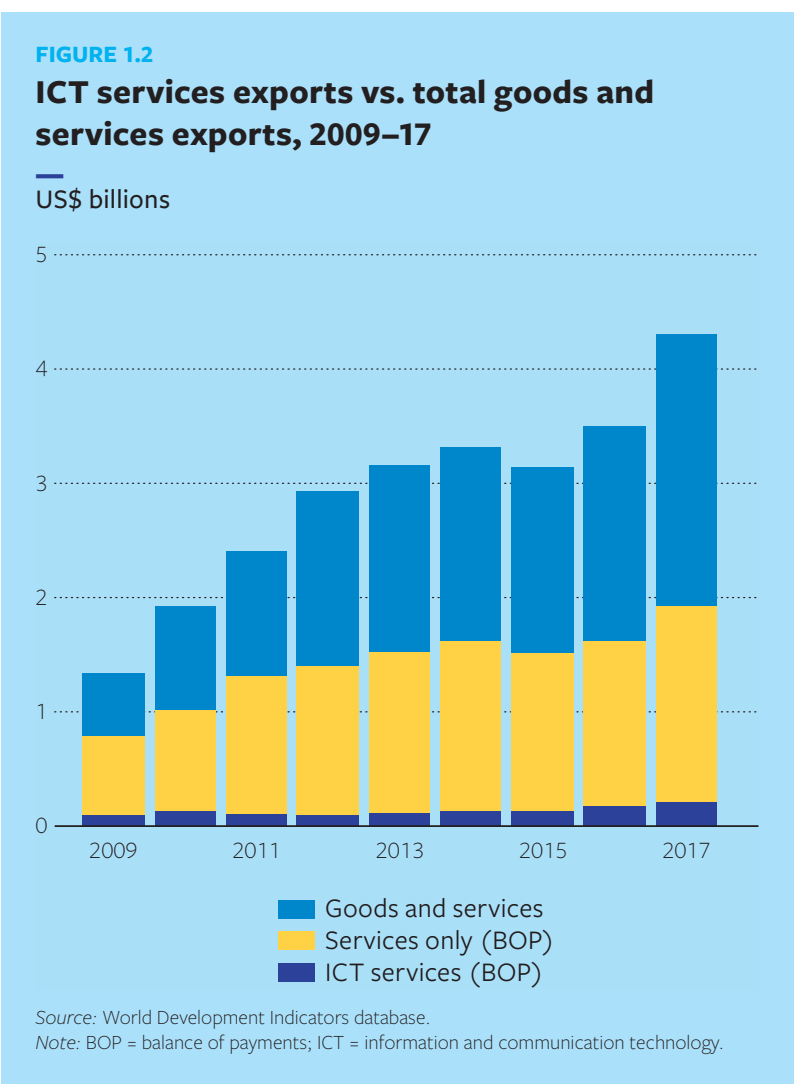

Software programming dominates Armenia's ICT services exports, but it has historically comprised outsourcing activities that are now trending toward automation. Armenia initially grew through low-cost outsourcing, notably customized software and web design (see figure 1.5). As noted in the World Development Report 2020: Trading in the age of Global Value Chains, global trends are toward a reduction in reliance on human resources to perform such functions and, even within Armenia, several firms have started to develop programs that automate software design services. As an example, the Armenian firm 1oWeb has developed a software that enables users to build WordPress websites rather than outsourcing design. Similarly, Renderforest, another Armenian company, uses artificial intelligence (AI) algorithms for animated video production.

Encouragingly, much of Armenia's "product and service solutions" activity (as opposed to outsourcing services) is already integrated into GVCs. Several global tech firms have operations in Armenia, with 38 percent of Armenia's ICT turnover coming from subsidiaries of foreign firms. The most high-profile of these are Synopsys, Mentor Graphics, and National Instruments, which together employ about 1,200 people in Armenia. This subsidiary model has been a strong source of growth, creating jobs, and technology and skills transfer (including managerial and soft skills). However, the value created in Armenia through these subsidiaries is not retained beyond salaries and overhead costs.

In recent years, a vibrant tech start-up culture has emerged, producing high-profile firms that have achieved global success. In the past few years, Armenia's tech start-up ecosystem has begun to flourish. Up to 200 start-ups have emerged that are supported by programs and organizations providing idea validation, acceleration services, seed investments, networking and coworking facilitation, business centers, government initiatives, and entrepreneur associations. As an example, PicsArt, valued at US\$250 million, has developed an image-editing and social-networking app that has been downloaded 600 million times. Another mobile app developer with its origins and development team based in Armenia, SoloLearn, has 1 million active monthly users globally.

Armenian firms are competing globally to deliver mass-market software and hardware solutions for a range of vertical applications. Armenia's software development community is designing solutions for a wide range of global vertical applications. Firms have targeted these activities and applications because they have low barriers to entry and solutions that can be readily developed and delivered remotely. Examples include human resources management software development, lifestyle mobile applications, banking software, social media software, and educational training kits. However, the same low barriers to entry that make the mass-market applications "attractive" also make them highly competitive globally.

In other cases, activities that Armenian firms can compete for are (necessarily) disconnected from other high-tech activities in the global value chain, limiting opportunities for upgrading or vertical integration. In a number of vertical applications, the reason that global firms are able to provide services "remotely" is because the activity is relatively disconnected from the rest of the value chain. This characteristic lowers the barriers to entry for that specific activity but makes it difficult for Armenian firms to upgrade or vertically integrate "adjacent" higher-value-added activities. In some cases, there are few other high-tech activities in the value chain to target for upgrading. For instance, several Armenian firms design and manufacture standalone educational electronics toolkits for schools and universities. While 
FIGURE 1.3

ICT services exports per capita, Armenia and regional high performer comparators, 1995-2017

$\bar{a}$. Armenia and regional comparators, US\$

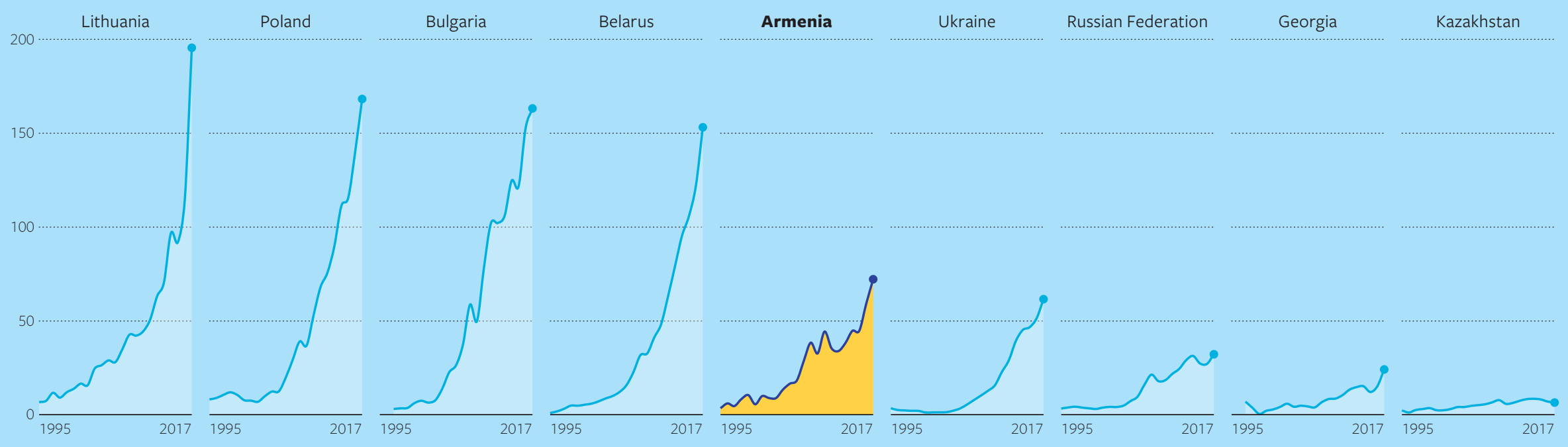

b. Armenia and High performer comparators, US\$

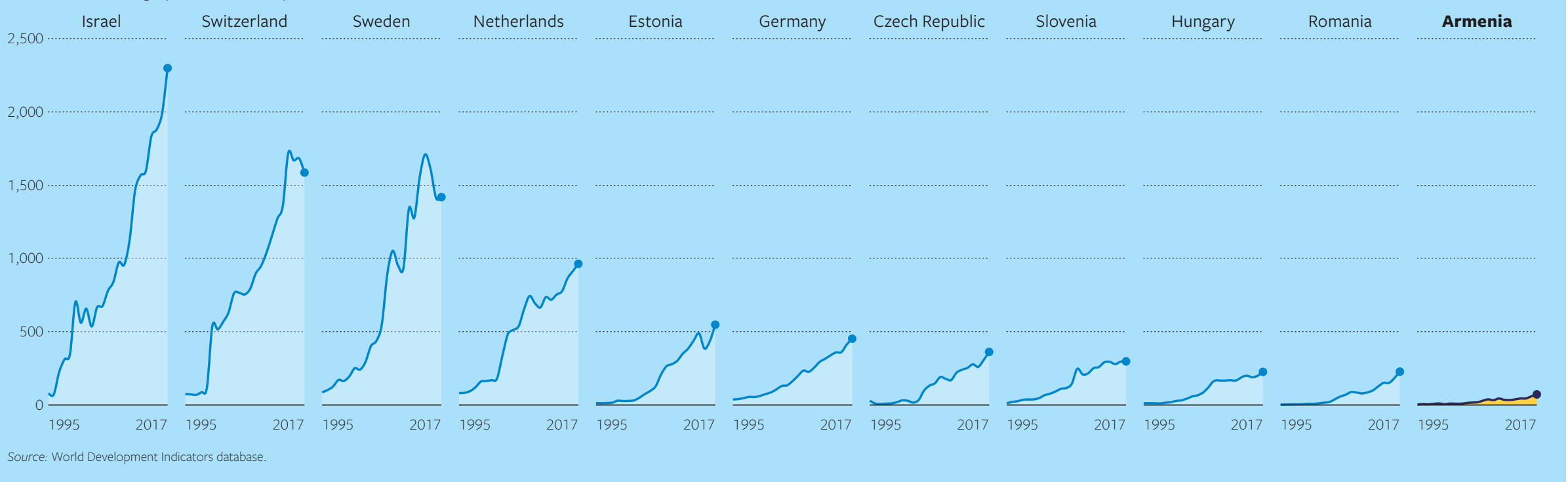

6 I ARMENIA'S CURRENT POSITIONING IN HIGH-TECH GVCS 
FIGURE 1.4

ICT exports per capita vs. CAGR of ICT services exports

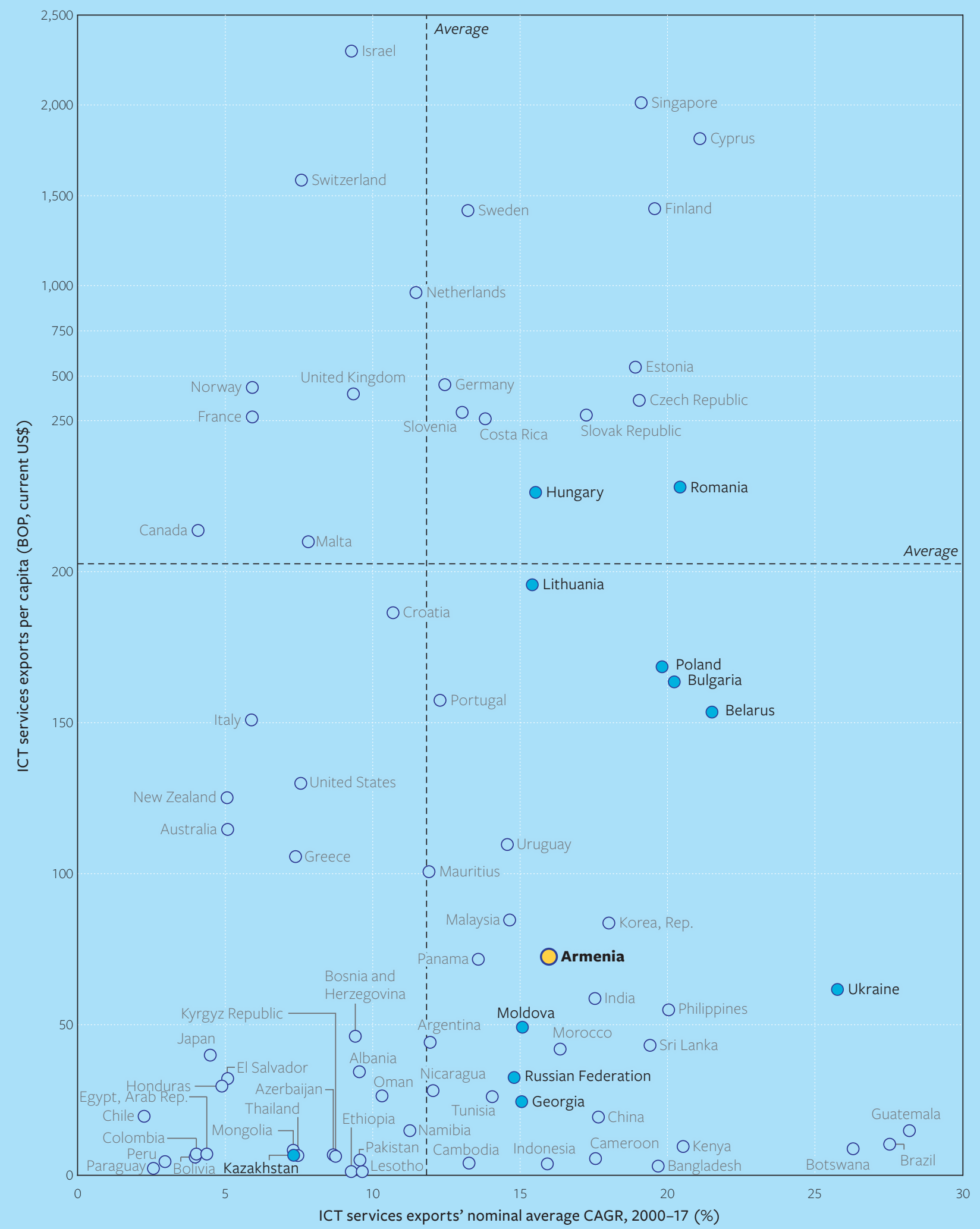

Source: World Development Indicators database.

Note: $\mathrm{BOP}=$ balance of payments; $\mathrm{CAGR}=$ compound annual growth rate. Regional comparator countries are highlighted in blue. 


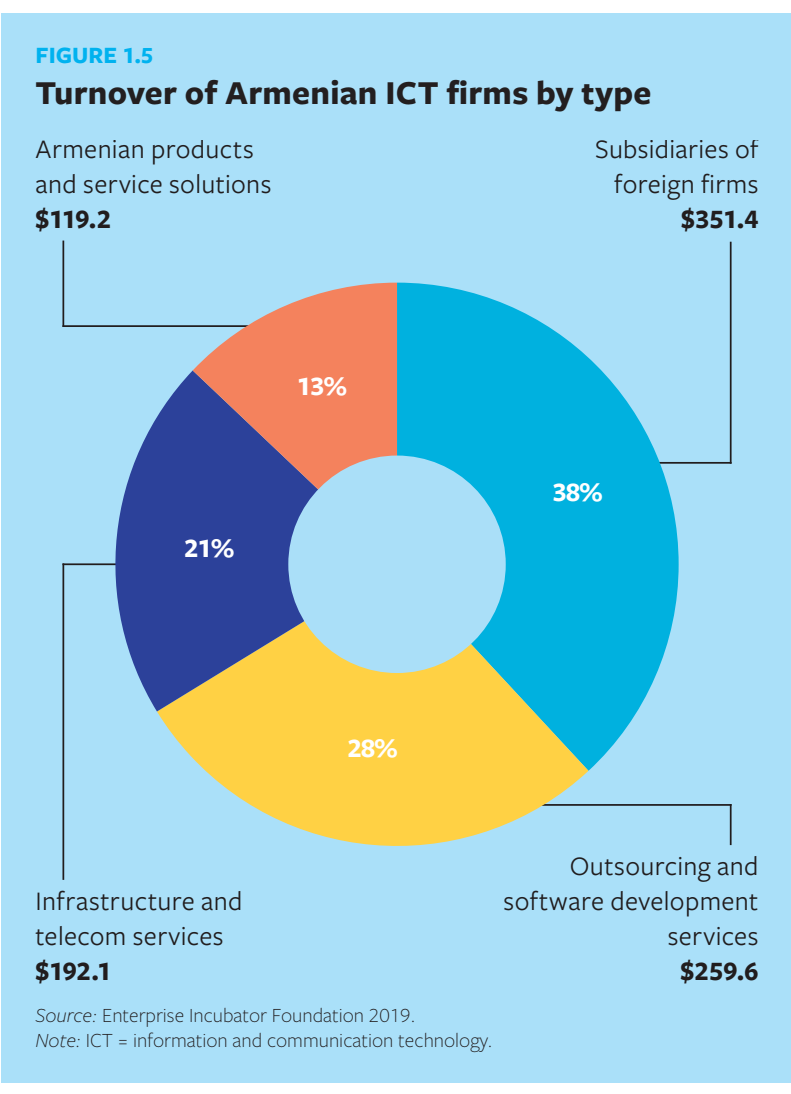

these firms were able to enter the market relatively easily, $^{2}$ they have found few opportunities to upgrade or expand their activities.

In addition, as the customer base for many of these vertical applications is far from Armenia, generating new business has proven difficult. Whether for business-to-business or business-to-consumer solutions, the broad base of end-users for Armenian software products and solutions is outside of Armenia. Indeed, there is a heavy bias toward U.S. markets and clientele. Entry into certain sectors has often been serendipitous, and several Armenian start-ups and small and medium enterprises have indicated that they have struggled to generate new business and expand their customer base. At the same time, many of Armenia's competitors are much closer to the end-user and may have a more intuitive understanding of their needs.

Continuing to compete for mass-market solutions will be increasingly difficult for Armenia as global competition from automation (and lower-wage countries) increase. While it is important to increase the value of Armenia's ICT services exports, the types of exports (and underlying activities therein) are equally important, particularly as some software development functions face threats from AI and machine-learning technologies (see section 2.2). At the same time, the World Development Report 2020: Trading for Development in the Age of Global Value Chains notes that developing countries stand the most to gain from the reduction in trade costs that arise from digital technologies (because they face the highest trade costs and biggest distortions), adding further competitive pressures.

The government can strategically support Armenia's migration toward activities that are catalyzed-rather than threatened-by emerging technologies. Armenia's tech community is poised to move beyond software development toward deeper participation in the development and commercialization of emerging and disruptive technologies. As discussed in chapter 2 , the government can facilitate this transition by strategically repositioning Armenia's ICT services providers in (a) activities that leverage Armenia's factor conditions (see section 2.1); (b) "adjacent" high-value-added technologies that build on the existing competencies of Armenian firms (see section 2.2); and (c) the development of nascent technologies while they are still accessible (see section 2.3). In addition, the regulatory ecosystem could be further strengthened (see chapter 3 ). These opportunities are discussed in the chapters that follow. 


\section{Chapter 2}

\section{STRATEGIC GVC REPOSITIONING OPTIONS FOR ARMENIA}

"High-tech sectors" is a vast grouping that in 2019 could describe virtually all vertical sectors. While discussions surrounding disruptive technology quickly turn to 3D printing, augmented reality, internet of things (IoT), blockchain, and artificial intelligence (AI), the so-called "traditional" sectors (e.g., automotive and pharmaceutical) are just as "digital" as Amazon. A widely shared 2017 infographic demonstrated that there were more lines of code in a modern high-end car (100 million) than in Facebook (61 million) (McCandless 2017). Even agriculture is no longer exempt; global positioning satellite guidance systems, sensors, robotics, control systems, farm management software, drones, and telematics are all frequently used to increase the quantity and accuracy of information available to farmers in order to maximize returns, increase efficiency of production, and reduce waste (Schmaltz 2017). Crucially, each one of these vertical sectors has its own value chain and its own determinants of competitiveness. This chapter identifies different strategies that could be employed by the government to position Armenia to deliver higher-value-added, tech-related activities in identified sectors. As sectoral development strategies rely heavily on strong private sector engagement and open public-private dialogue, Armenia's tech community may also take interest in this analysis. It is important to note that the sectoral examples highlighted in the following sections are rele- vant at the time of writing. As the technology revolution continues, some of the presented examples may "expire," giving way to new opportunities in other emerging technologies.

2.1

\section{LEVERAGING ARMENIA'S FACTOR CONDITIONS TO DEVELOP GLOBAL NICHE ACTIVITIES}

\section{Armenia's software and hardware development community currently competes to deliver} mass-market applications. Currently, Armenia's software and hardware development community targets highly saturated global vertical sectors where: (a) Armenian firms have little or no comparative advantage, (b) opportunities for upgrading and vertical integration are limited, and (c) building a customer base is reliant on proximity. Examples include human resources management software development, lifestyle mobile applications, banking software, social media software, and educational training kits.

\section{Armenian firms may do better to leverage} armenia's factor conditions to target global niche vertical applications. Armenia has several special characteristics (e.g., geology, population characteristics, geography, and industrial history) that could make it 
particularly conducive to developing solutions for certain vertical applications. With the support of strategic policies and investments, there is an opportunity for Armenian firms to target these niches and build stronger offerings to better compete in global markets. This chapter describes three such global niches that Armenia could develop.

A strategic investment promotion strategy could attract global firms in these niches, "localizing" the global market. Where there is a strong "local" component to Armenia's comparative advantage in a particular niche (for instance, those related to geology or population characteristics), it might be advantageous for foreign firms to locate in Armenia to take advantage of certain factor conditions for research, development and testing. The Armenian government has the opportunity to orient investment promotion policies toward these niches. In so doing, the government could "bring the global market to Armenia," reducing information asymmetries and encouraging close cooperation and collaboration between global firms and local innovators and start-ups.

\section{BIOTECH AND DIGITAL HEALTH}

Armenia's population has distinctive health characteristics that could be valuable to global biotech development. The incidence and mortality rates of cancer, cardiovascular, and neurodegenerative diseases is unusually high in Armenia. Out of 183 countries, Armenia has the second-highest cancer mortality rate in the world for both men and women (WHO 2014; Bray et al. 2018).

As the government codifies these health characteristics through its digital health record system, armenia's software development community can build on the resulting digital assets. Armenia recently became one of the few countries in the world with a nationally centralized digital health record system (ArMed). ArMed was designed not only to improve access to and quality of health care but also to facilitate medical education and research. In addition, there has been much discussion about the Armenian Genome Project (AGP) over the past 18 months. The AGP is significant due to the presumed genetic homogeneity ${ }^{3}$ of the Armenian population. Armenia's software development community can integrate AGP and ArMed data to design solutions for global markets as demand for digital health care solutions widens to include a variety of users such as physicians, insurance companies, research centers, and pharmacies.
With an appropriate regulatory framework, Armenia can position itself as a global hub for digital health, biotech development, and bioscience research. Building on these factor conditions, Armenian firms could expand their global value chain (GVC) participation by moving from digital health to biotech devices and associated software solutions. This could include (hardware and software) design of implantable devices (e.g., cardioverter defibrillators), wearable devices (e.g., continuous glucose monitors) and IoT solutions (e.g., sensors for smart watches and mobile applications). In so doing, Armenia could become a global hub for digital health technologies, attracting foreign direct investment and international research institutions. (This opportunity for Armenia is discussed in depth in the case study on ICT services for digital health and biotech applications, page 22.)

\section{CLEANTECH}

Armenia's mining history has resulted in significant ongoing air and water pollution and land contamination. Mining is Armenia's dominant export sector. Copper ore alone accounted for 35 percent of Armenia's exports in 2017, while aluminum foil, raw copper, gold, and diamonds accounted for 16.8 percent.

Unfortunately, mining practices continued from the Soviet era have resulted in significant ongoing air and water pollution, in addition to extensive land contamination through heavy metal (e.g., lead and arsenic) residue. In addition, wastewater spillages and leakages have led to contamination of downstream waterways.

The cleantech industry could mitigate some of these environmental damages. In addition to alternative energy solutions, cleantech solutions include products and services that mitigate negative environmental impacts. This encompasses a wide range of technologies in reclamation and soil and water treatment including biofiltration, sediment capping, soil flushing, dredging, and chemical stabilization. The industry is supported by small and medium innovators and researchers as well as large multinationals to respond to topographical, environment and regulatory challenges.

Not only does the global cleantech industry have very large growth potential, small and medium enterprises (SMEs) in middle-and low-income countries will have critical roles to play over the next decade. The potential for global cleantech is huge. Investment across 15 clean technology sectors in 145 developing countries is expected to top US\$6.4 trillion over the next decade (World Bank 2014). Critically, 
over one-third of the total investments (about US\$3.59 trillion) will be directed toward water and wastewater solutions. This market is not reserved for large multinational companies only. For SMEs in middle- and low-income countries, US\$1.6 trillion of this market is potentially available (see figure 2.1).

Rather than relying on the local mining industry as a market, Armenia can become a concentrated research, development, and prototyping testbed for global cleantech firms. Based on current investment in environmental risk solutions, it is unlikely that Armenia's local mining industry can form the cornerstone of a cleantech market. Similarly, Armenia's public agencies may not yet be in a position to improve or enforce environmental regulation in this field. However, global high-tech firms and research and development (R\&D) institutions can still utilize Armenia's factor conditions as a concentrated solutions-development and prototyping testbed for cleantech solutions to serve global markets. In Eastern Europe, close to US\$30 billion of the clean technology market for wastewater solutions are particularly accessible to SMEs (see figure 2.2). By attracting foreign firms to locate themselves in Armenia, the government can create a local market for

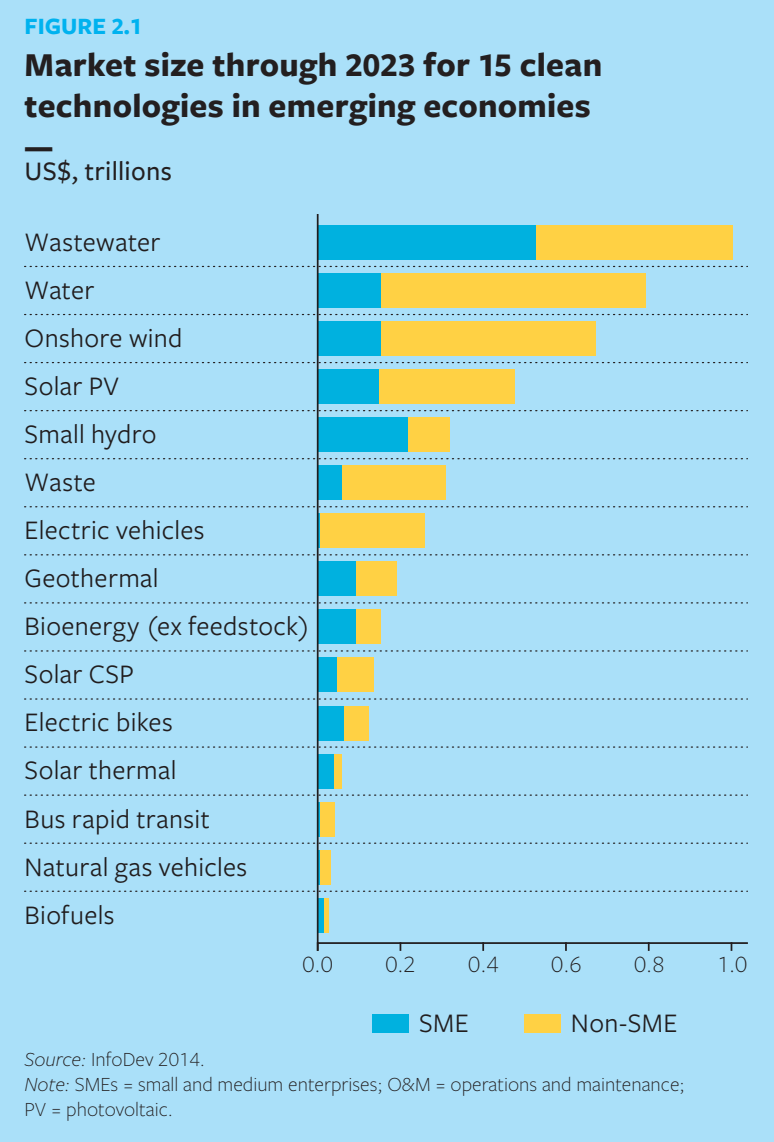

\section{FIGURE 2.2}

\section{Size of the clean technology market accessible to SMEs in Eastern Europe}

$\overline{U S} \$$ billion

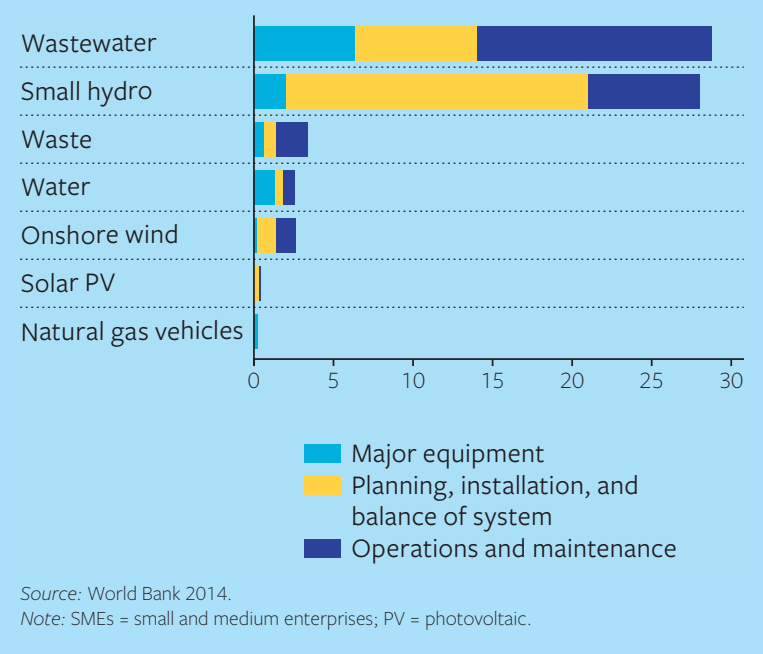

upstream, higher-value-added research and development activities, building local capacity and expertise and facilitating innovation in this field. Strengthening Armenia's foreign direct investment (FDI) laws and investor "aftercare" would be key to success in this kind of development strategy.

\section{EARTHQUAKE ENGINEERING}

Located on the high activity Alps-Himalaya seismic zone, Armenia is prone to earthquakes and related hazards. As a result of compression between the Arabian and the Eurasian tectonic plates, Armenia is home to several geological faults and has a long history of seismic activity. This has included large and destructive earthquakes such as the 1988 Spitak earthquake, which killed almost 25,000 people, injured 15,000 people, and left 517,000 people homeless.

\section{Mitigating seismic risk has become a high-tech} global industry. Structural engineering, geotechnical engineering, advanced materials engineering, and nonengineering fields such as catastrophic risk modeling and insurance markets all form part of a growing global industry that targets reduced loss of life and property damage during seismic events. In reflection of this, the geotechnical instrumentation and monitoring market is expected to grow to US\$5 billion by 2024 (Markets and Markets Research 2019), while the market for seismic reinforcement materials is projected to hit US $\$ 40$ billion by 2025 (Pulidindi and Chakraborty 2019). 


\section{Although innovation hubs are concentrated in} high-income economies, middle-income countries have leveraged earthquake preparation programs to develop local capacity. Global innovation hubs are concentrated in rich, earthquake-prone economieslike Italy, Japan, New Zealand, and the United States-where there is a public and private sector market for risk-mitigation technologies and solutions. In addition, other countries like Germany and the U.K. (neither of which experience high seismic risk) have become global hubs for catastrophic risk modeling due to their status as global insurance centers with access to high-performance computational infrastructure. However, middle- and low-income countries outside of these hubs have been able to leverage government earthquake preparation programs to develop local capacity in seismic risk assessment and structural retrofitting. Examples include Bulgaria, India, Pakistan, and Romania, which have wide structural retrofitting programs, and Turkey, whose reconstruction program has also contributed to a local "earthquake engineering" industry supported by geophysicists, structural engineers, geologists, and economists. ${ }^{4}$

The Armenian government can strategically leverage its own earthquake preparation programs and building code regulations to develop Armenia's local earthquake engineering capacity. An expansion of programs like the government's Seismic Safety Improvement Program (which aims to retrofit and build earthquake-resilient schools) could similarly help to develop geotechnical expertise in Armenia that could serve the Caucasus more widely. Note that stronger building code regulation, inspection and enforcement would be key to catalyzing the earthquake engineering industry. In addition, strengthening these policies could also support the development of a private sector led insurance market; currently, the information asymmetry relating to the vulnerability of physical infrastructure in Armenia has made it difficult for this important sector to flourish.

Targeted R\&D investments, policies, and incentives would be key to the development of geotechnical services in Armenia. Beyond earthquake early warning research, Armenia can leverage its location to develop new research in fault zone and earthquake geology fields (e.g., paleoseismology, tectonic geomorphology, and seismic imaging) as well as earthquake processes and effects (e.g., crustal deformation, ground motion, and ground failure). In Armenia, this activity is dominated by public institutions (such as the National
Service for Seismic Protection, the Institute of Geophysics and Engineering Seismology, and the Institute of Geology) and other nonprofit institutions, though strong private institutions are starting to emerge (e.g., GEORISK Scientific Research Company). Private sector-led innovations could be further catalyzed by targeted research policies including applied research grants, competitive research collaborations, competitions, and R\&D tax credits.

2.2

\section{UPGRADING TO “ADJACENT" HIGHER- VALUE-ADDED ACTIVITIES}

\section{GVC upgrading can come through vertical integra-} tion of value-chain activities that are adjacent to Armenia's existing ICT services exports. Armenia's policy makers can examine the high-tech activities that Armenian firms already perform well and implement targeted programs to support vertical integration into "adjacent" higher-value-added activities. This report examines opportunities for upgrading to (a) AI research and engineering (building on Armenia's competence in mathematics and computer science) and (b) semiconductor design (building on Armenia's strong foothold in electronic design automation).

\section{AI PURE RESEARCH}

Because pure AI research (the creation of new algorithms) relies on advanced mathematical research techniques, mathematicians and statisticians are in high demand in this field. AI algorithms can be written in most programming languages, including Java, Python and R. As a result, there is a widespread misconception that pure $\mathrm{AI}$ algorithm research is a field of computer science or software programming. In fact, $\mathrm{AI}$ algorithm development uses advanced mathematical research techniques (e.g., calculus, linear algebra, probability, statistics, and heuristics) that are more common in natural science disciplines such as physics, mathematics, and econometrics. As the AI market continues to expand, scientists and researchers with deep understanding in these fields are in increasingly high demand.

Armenia has strong global comparative advantages in mathematics and natural sciences-even more so than in ICT. In 2017, Armenia produced 0.38 per 1,000 tertiary graduates from ICT-related courses. In the same year, Armenia produced 0.6 per 1,000 


\section{FIGURE 2.6}

\section{NSMS vs. ICT}

Number of tertiary graduates per 1,000 people, $2016 / 17$

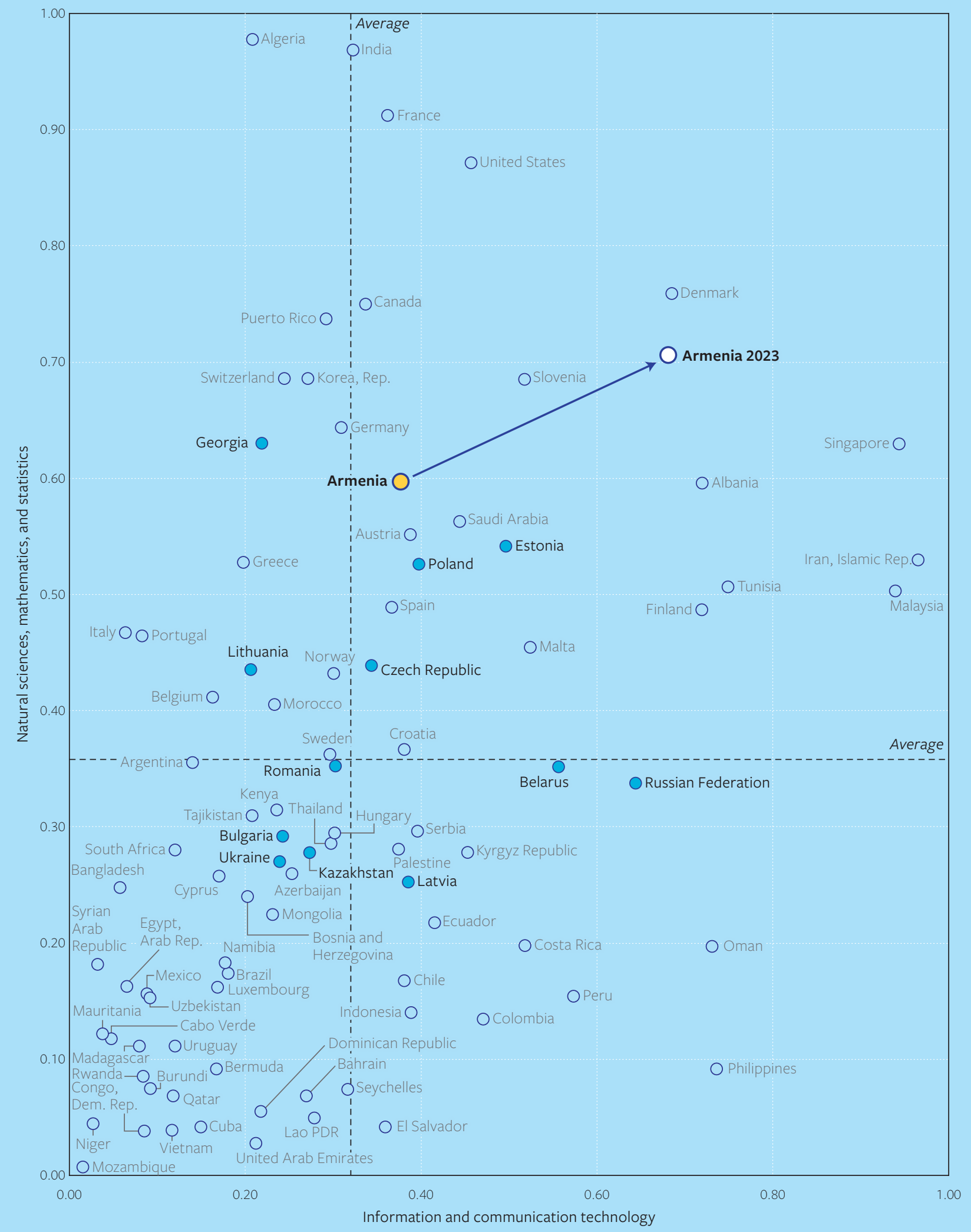

Source: United Nations Educational, Scientific and Cultural Organization's Institute for Statistics database.

Note: NSMS = natural sciences, mathematics, and statistics; ICT = information and communication technology. Armenia 2023 estimation assumes: (a) the distribution of bachelor's degree programs in 2022 corresponds to the distribution of bachelor's degree enrollments by program in 2018; (b) the ratio of tertiary to bachelor's graduates remains consistent; and (c) future tertiary graduate figures are a function of current primary and secondary enrollment figures. 
graduates in natural sciences, ${ }^{5}$ mathematics, and statistics (NSMS). In both measures, Armenia outperforms global averages (0.32 in ICT and 0.36 in NSMS) and many of its regional competitors (figure 2.6). Moreover, between 2016 and 2018, the share of bachelor's degree students graduating from NSMS programs grew by 50 percent. Tertiary graduates from mathematics and statistics programs alone grew by 68 percent. Based on current projections, ${ }^{6}$ by 20239.7 percent of Armenia's bachelor's degree students will graduate from NSMS programs. (Armenia's projected position is also shown in figure 2.6.)

\section{Mathematics and natural sciences curricula in} Armenia are current. Industrial technology has evolved at a relentless pace over the past 50 years. This dynamism has been particularly challenging in applied science and engineering courses where graduates must be cognizant of the latest technology breakthroughs, conversant in up-to-date industry tools, and display the necessary soft skills and commercial acuity. Globally, mathematics and natural science disciplines have not advanced at the same pace as applied science and engineering courses. As a result, course content for these fields in Armenian teaching institutions has been able to remain current.

Until recently, commercial opportunities for mathematicians in Armenia were few. After the collapse of the Soviet Union, many of the institutions that employed Armenia's mathematicians and physicists disappeared. Job opportunities for mathematicians wishing to continue to work in theoretical research and related fields are almost entirely confined to universities and teaching institutions where the quality of $\mathrm{PhD}$-level research has been called into question. As a result, promising graduates and researchers have left Yerevan to work in global research institutions.

Unlike in several pure research disciplines, competitiveness in AI pure research does not have high capital investment requirements. Traditionally, the potential for individual researchers and micro institutions to thrive has been constrained by the need for costly laboratory equipment. In the area of applied physics, for instance, leading universities and research institutions boast cleanroom facilities, microscopy and reflectometry equipment, processing equipment (including deposition, etching, and nanolithography equipment), and ultra-high vacuum equipment. This is not to mention large-scale facilities for research into condensed matter, materials science, and particle physics. In contrast, AI algorithm researchers need only an internet connection. Where supercomputers are needed-for example, for training more complex neural networks-they can be accessed remotely.

Armenia already has some nascent activity in AI. There is already some activity in pure AI research in Armenia. Several start-ups support their own in-house AI development teams for AI-enabled functionality. In addition, YerevaNN, a nonprofit computer science and mathematics research lab, recently published its first peer-reviewed paper in the Nature Scientific Data journal, and another paper is soon to be presented in a workshop organized by the Association for Computational Linguistics. On top of this, a number of Armenian physics professors have published papers relating to AI.

However, a lack of post-doctorate training in AI in Armenia is leading to a brain drain and undermining local investment opportunities. The American University of Armenia offers a bachelor of science program in data science, and the Yerevan State University $^{7}$ and the Slavonic University both have AI-related master's programs. However, there are no AI $\mathrm{PhD}$ programs in Armenia. Without a PhD program, the industry will struggle to grow as promising researchers (and firms that draw from this research) look outside Armenia for resources. There is already evidence of this; PicsArt, an Armenian firm (headquartered in the United States) has its own in-house AI development team based in Yerevan. However, in October 2018, PicsArt opened an AI lab in Moscow with the express purpose of leverage the AI talent pool emerging from the Moscow Institute of Physics and Technology (MIPT). PicsArt intends to enter into R\&D partnerships with the MIPT to further develop PicsArt's existing AI-based features, such as visual transformation, behavior prediction, and content recommendations.

In addition, corruption has undermined the integrity and quality of Armenia's PhD programseven in natural sciences. A 2018 report by the Organisation for Economic Co-operation and Development's Anti-Corruption Network for Eastern Europe and Central Asia (ACN) discusses the prevalence of corruption and corruption risks in the higher education system in Armenia. Using official documents, questionnaires, perception surveys, and third-party reports by research organizations, the monitoring team concluded that corruption is a widespread problem in Armenian higher education (ACN 2018). Vulnerable 
areas include university management, human resource policies, student assessment, licensing, and accreditation. The assessment also specifically highlights post-graduate education as an area where gaps in legislation and institutional structures have facilitated abuse. These risks are acknowledged by the Armenian government, which has specifically targeted corruption in the Anti-Corruption Strategy 2015-18. (The AntiCorruption Strategy 2019-22 is still in draft.) As a result of these strategies and consultations between government and civil society, a Programme for Anti-Corruption Measures in Education was agreed upon in early 2018. However, the ACN's assessment is that only three of 12 countermeasures were funded, and that only five of the 12 countermeasures are mandatory. Civil society and research organizations have also expressed concerns that the countermeasures are not thorough enough.

Low research quality and a lack of replenishment in the PhD education system will continue to be issues as long as professor salaries remain below the market rate. Professors, like graduates and researchers, can command higher salaries in higher-income countries. However, this common phenomenon is exaggerated in the case of university faculty. A 2012 study by the Center for International Higher Education at Boston College and the Laboratory for Institutional Analysis at the National Research University Higher School of Economics in Moscow showed that even when comparing purchasing power parity rather than real exchange rates, public higher-education faculty members receive very low salaries (Altbach et al. 2012). Armenians received the lowest compensation of the 28 countries in the study (figure 2.7). This issue is compounded by the fact that, whether in Armenia or abroad, faculty can often demand higher salaries in the private sector. As a result of these market failures, would-be professors and supervisors are not staying in research, so the $\mathrm{PhD}$ system is not replenishing itself.

In addition, with a stronger AI research base, Armenia's software programming and computer science community could also build a strong offering in AI engineering. AI pure research refers to the research and development of new AI algorithms that are designed to address hypothetical problems. AI engineering refers to the manipulation and adaptation of existing algorithms to "real-world" applications. Data science and software programming are core components of AI engineering. With a critical mass of researchers and new algorithm development, Armenia's

\section{FIGURE 2.7}

Monthly average salaries of faculty in public universities in 28 countries, 2012

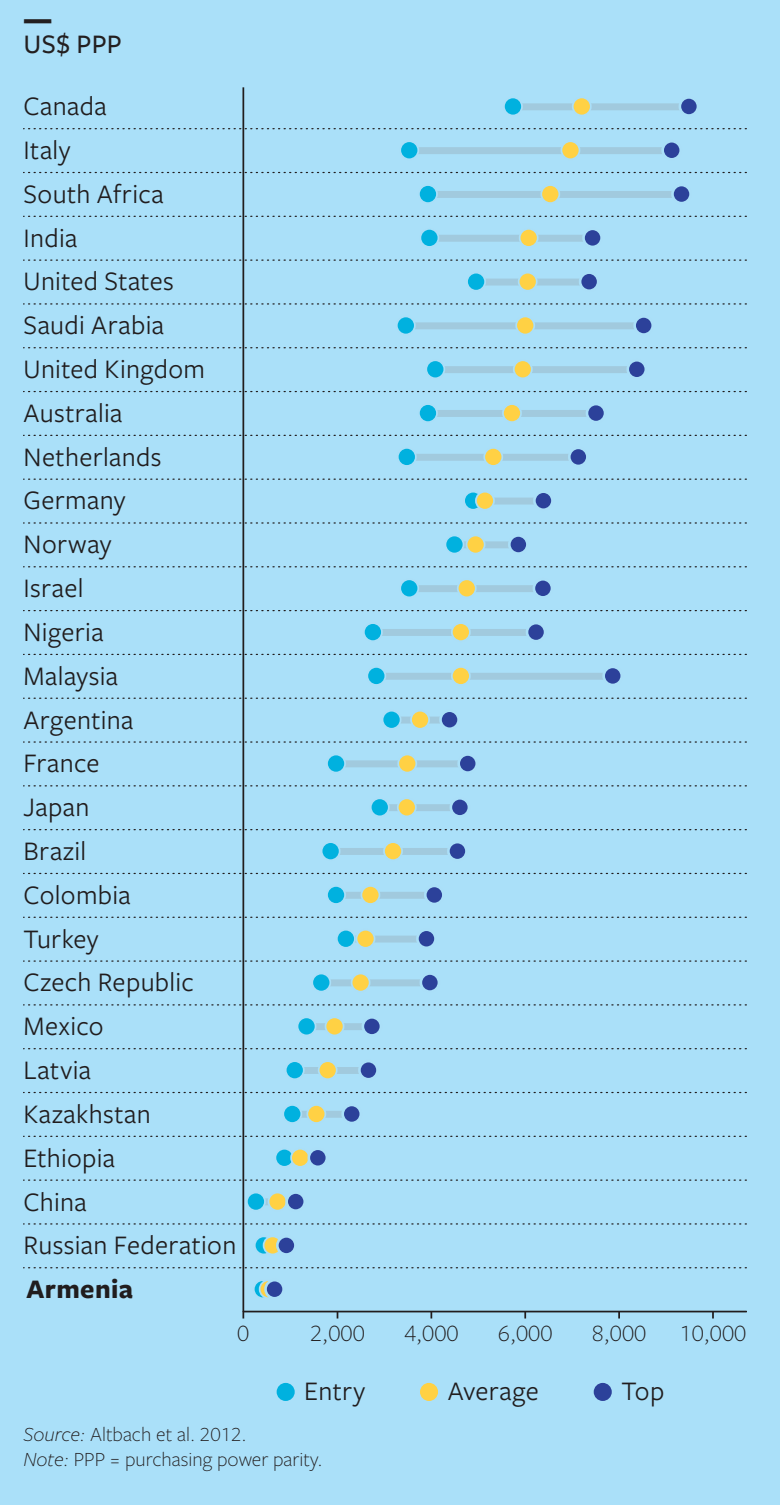

already well-developed software programming community could build a strong offering in AI engineering solutions. Already, an Armenian consortium, YEA Engineering, is developing a platform for verification and validation of advanced driver-assistance systems, as well as for end-of-line testing in vehicle-in-the-loop environments.

However, moving from AI research to AI engineering is contingent on being given access to proprietary data and being able to protect those data. In AI pure research, new algorithms are deliberately developed using publicly available data so that they can be peer reviewed in international fora. However, to apply and adapt an AI algorithm to a 
specific problem (AI engineering), AI teams need real-world client datasets. Few companies in Armenia have large volumes of digital data (the exceptions being companies such as GG-Taxi and U-Com). Foreign companies will increasingly demand that Armenian firms comply with global information security and management, such as the ISO/IEC 27000-series. Compliance with Europe's General Data Protection Regulation 2016/679 will also influence the ability of Armenian firms to train neural networks on data from private companies within the European Union (EU).

The Armenian government can implement policies to effectively collect, digitize and share both administrative and statistical data for application development. The government may be able to plug the gap in available data for application development and fostering research. Where regular data collection is already taking place, policies and practices differ significantly between ministries, and there is no clear policy or necessary infrastructure for efficient data exchange in the government-to-government, government-to-business, and government-to-consumer domains. There is an opportunity for the Armenian government to standardize the collection and digitization of both administrative and statistical data. The government can also design policies relating to the classification, formatting, storage, and exchange of these data. More generally, there are no standardized mechanisms, policies, or capacities for data-driven policy making or encouraging regional cooperation (specifically with the Eurasian Union). To harness both internal and international development opportunities offered by the global data economy, Armenia should in parallel develop data policies (including data management, cybersecurity, and other policies), amend its legal framework accordingly, and create future-proof data infrastructure (broadband networks and data centers).

\section{SEMICONDUCTOR DESIGN}

Two of the three dominant global electronic design automation (EDA) firms have operations in

Armenia. EDA software is used by the semiconductor industry to design chips. ${ }^{8}$ In 2004, Synopsys entered Armenia through its acquisition of Leda Design and Monterrey Arset, both of which were engaged in EDA software design. Synopsys is now Armenia's largest ICT employer; with 800 employees, it is the largest of Synopsys' offices among its 12 global subsidiaries.
Another EDA firm, Mentor Graphics (part of Siemens), also entered the Armenian market in 2008 by acquiring the assets of Ponte Solutions Inc. The most recent firm to set up EDA operations in Armenia-Xilinx, Inc.-will not be doing so through acquisition. Instead, Xilinx has begun a training and employment program to recruit software engineers for its Armenia operations. This is significant because Xilinx will be producing EDA software for field programmable gate arrays (FPGAs), as opposed to standard chips. FPGAs are dynamically programmable, offering higher performance and versatility for a multitude of applications. Xilinx's decision to locate is operations in Yerevan is testament to Armenia's core capability in EDA.

Globally, the EDA industry is highly consolidated-start-ups and small firms are rare. Due to the need for synchronization and standardization across the sector, the EDA industry is highly consolidated. In 2017, just four companies captured about 68 percent of global revenues: Synopsys (valued at US\$19.37 billion), ${ }^{9}$ Cadence Design Systems (valued at US\$19.26 billion), ${ }^{10}$ Mentor Graphics (valued at US\$4.5 billion), ${ }^{11}$ and ANSYS Inc (US\$17.58 billion). ${ }^{12}$

Semiconductor chip design-a higher-value-added activity-relies on the same core skillset as EDA software design but is better suited to start-ups and small firms. EDA software is used by firms across the semiconductor industry to design chips. Granted that some of these companies are very large (Intel, $\mathrm{ARM}$, and Samsung being among the largest), but there is a greater degree of fragmentation than in the EDA industry. "Fabless" semiconductor firms (firms that design and sell complete chipsets but outsource their fabrication) and intellectual property (IP) core developers (firms that design IP cores-the building blocks of semiconductor chips) ${ }^{13}$ are often small- or even micro-sized firms.

The potential of start-ups and small firms to spin off from Armenia's EDA firms and compete in semiconductor design may depend on the vertical application. Three broad applications have emerged in the semiconductor industry in recent years: (a) ultra-high-performance, high-volume applications (e.g., consumer electronics, $5 \mathrm{G}$ networks and cloud computing); (b) sensors for IoT proliferation (these do not require "latest-node" ${ }^{14}$ technology, but it is a high-volume, low-margin segment demanding little differentiation); and (c) boutique applications that do not require high-volume production but instead demand tailored, customized solutions (e.g., automo- 
tive). Each segment experiences different competitive pressures and therefore exhibits different capital investment requirements, economies of scale, and barriers to entry (figure 2.8).

Semiconductor and IP design are dominated by larger firms in both ultra-high-performance, high-volume applications and sensors for IoT proliferation. Faster switching speeds and higher performance are still key drivers in consumer electronics (e.g., smartphones, tablets, and laptops) and cloud computing infrastructure. As a result, the industry has seen more vertical integration in recent years in applications where high costs can be amortized, and large electronic device manufacturers (e.g., Google, Amazon, Huawei, and Panasonic) have started to design their own chips for their own specific use. In the meantime, Samsung Electronics has remained wholly vertically integrated throughout the industry's evolution and performs all functions across the value chain from design to manufacture. In the case of IoT, for devices to communicate with one another, there must be some degree of homogeneity between them. As a result, the industry favors high-volume, undifferentiated, low-margin production.

There could be an opportunity for Armenian firms in boutique vertical applications that do not require high-performance, high-volume production but instead demand tailored, niche solutions. Semiconductor applications where production at the latest "process node" is less important and the emphasis on volume is reduced are more accessible to smaller firms in Armenia. Smaller IP core designers and semiconductor designers are today designing bespoke solutions for specific vertical applications (for example, autonomous vehicles or health) or nicheenabling technologies. Similarly, there are critical enabling technologies that are still niche but have wider industry applications, for example, AI accelerators and ultra-low-power solutions. Semiconductor and IP design firms offering vertical applications differentiate themselves through their understanding of the client base and downstream hardware and software technology limitations. In addition, operational budget and cashflow must be conducive to the product development lifecycle of the target industry. Success in this area may depend on the development of strong and dynamic local (or regional) tech industries (see section 2.2).

However, venture capital requirements in semiconductor design are far higher than the venture capital available in Armenia today. Cisco, through its acquisition of the U.S. firm Memoir Systems, is one of the only global vendors with operations in Armenia. Although Cisco's IP design activity represents an important milestone for the country, there is a long way to go to convert Armenia into a true research and design hub for semiconductor technologies. According to Gartner, a research company, a latest-node semiconductor costs US\$271 million to design. Start-ups that are able to raise the necessary capital rarely see returns for two or even three years, as the design process is lengthy. Less advanced semiconductors can be cheaper to design, but even 28 nanometer semiconductors (2010 technology) cost US\$30 million to design in 2018 (Lapedus 2018). "Soft IP" only-the initial stage of IP core developmentcosts about US\$10 million, and "Hard IP," the second stage of IP core development requiring access to silicon labs for testing and verification, can cost about US\$50 million (note that sending the first IP core for testing can cost about US\$10 million in itself). However, Armenia's current available investment capital is only about US\$15 million, and therefore any gains in semiconductor design would rely heavily on foreign investment capital or an aggressive ramp-up in venture capital funding in Armenia.

Additionally, Armenian firms that can access funding may struggle to collaborate with leading international firms because the semiconductor industry is subject to controls under the Wassenaar Arrangement (WA). The WA is an agreement between 42 participating states to control exports of all items listed in the List of Dual-Use Goods and Technologies and the Munitions List to further international security and stability. The semiconductor industry receives some of the most scrutiny and limits exports of not only semiconductor systems, equipment and components but also test, inspection and production equipment, materials, and software. Furthermore, the restrictions go beyond export of tangible goods and include cooperation, collaboration and sharing information. While participating states can freely trade in these goods and services among one another, exports to other countries are controlled depending on their group designation (A, B, C, D, or E) ${ }^{15}$ as defined by the U.S. Department of Commerce. Because Armenia is a group D country, WA participating states wishing to collaborate and share listed technologies with Armenian firms need to first obtain export licenses. This obligation increases costs and 
FIGURE 2.8

Capital expenditure requirements, firm consolidation, and barriers to entry are different in different segments

\section{GROUP 1}

HIGH-VOLUME, HIGH-PERFORMANCE MASS-MARKETS

For example, consumer electronics, $5 \mathrm{G}$ networks and cloud computing

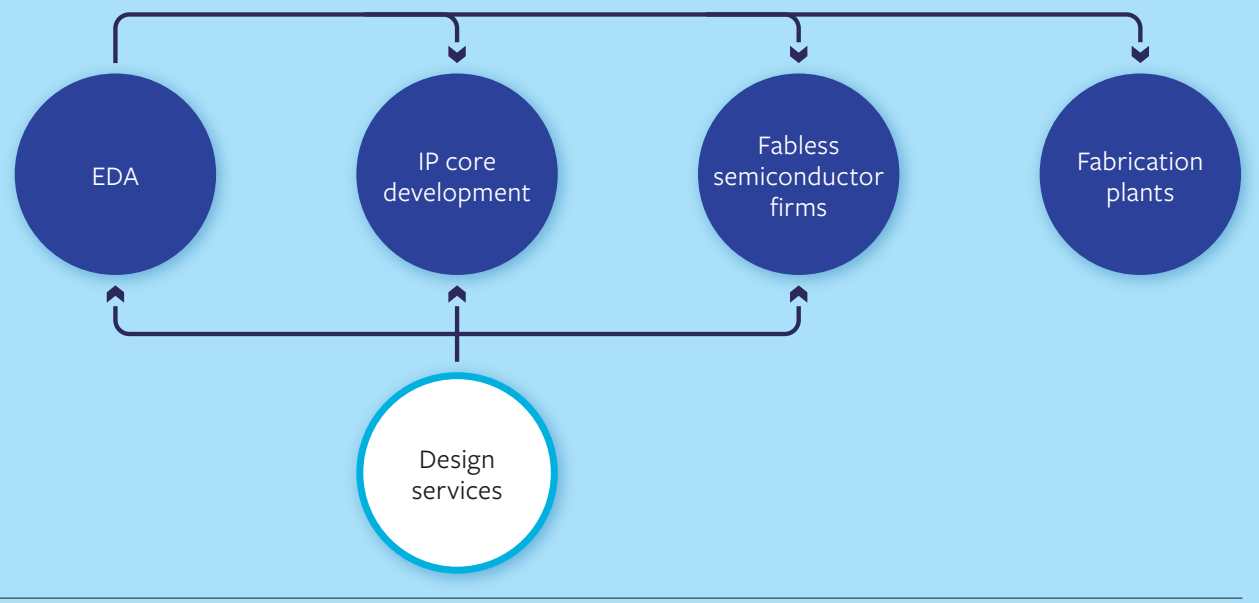

$\overline{\text { GROUP } 2}$

\section{SENSORS FOR IOT} PROLIFERATION

Undifferentiated design to facilitate high-volume, low-margin production

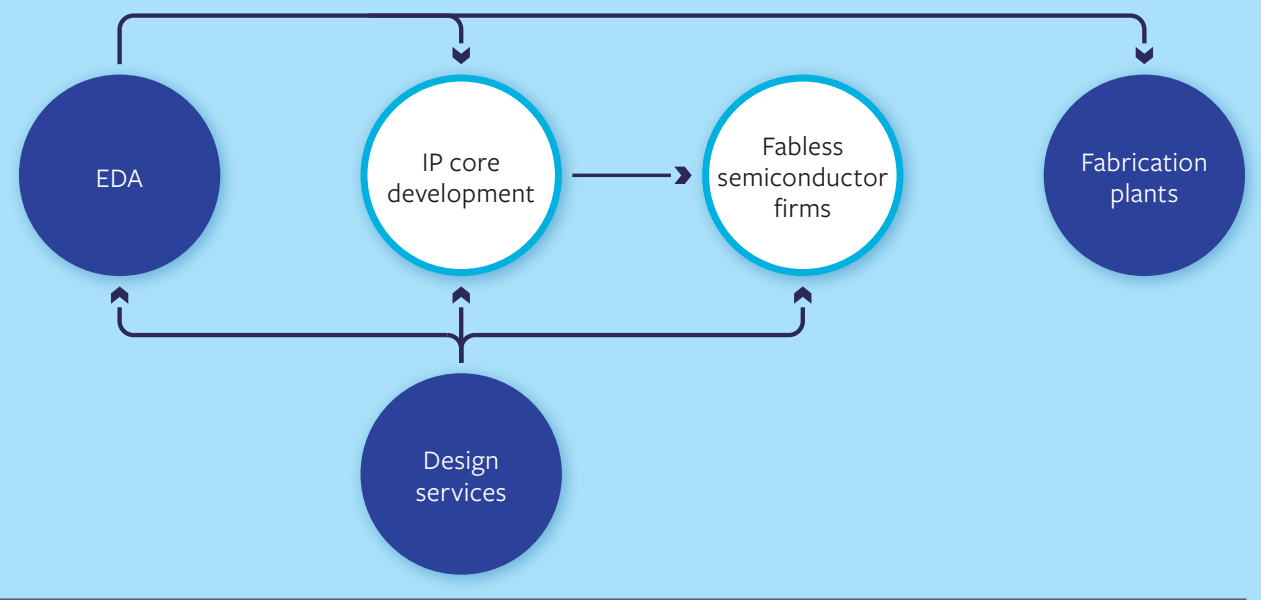

GROUP 3

A. NICHE VERTICAL APPLICATIONS

For example, autonomous vehicles or health

\section{B. NICHE-ENABLING} TECHNOLOGIES

For example, AI

accelerators, ultra-low-

power solutions.

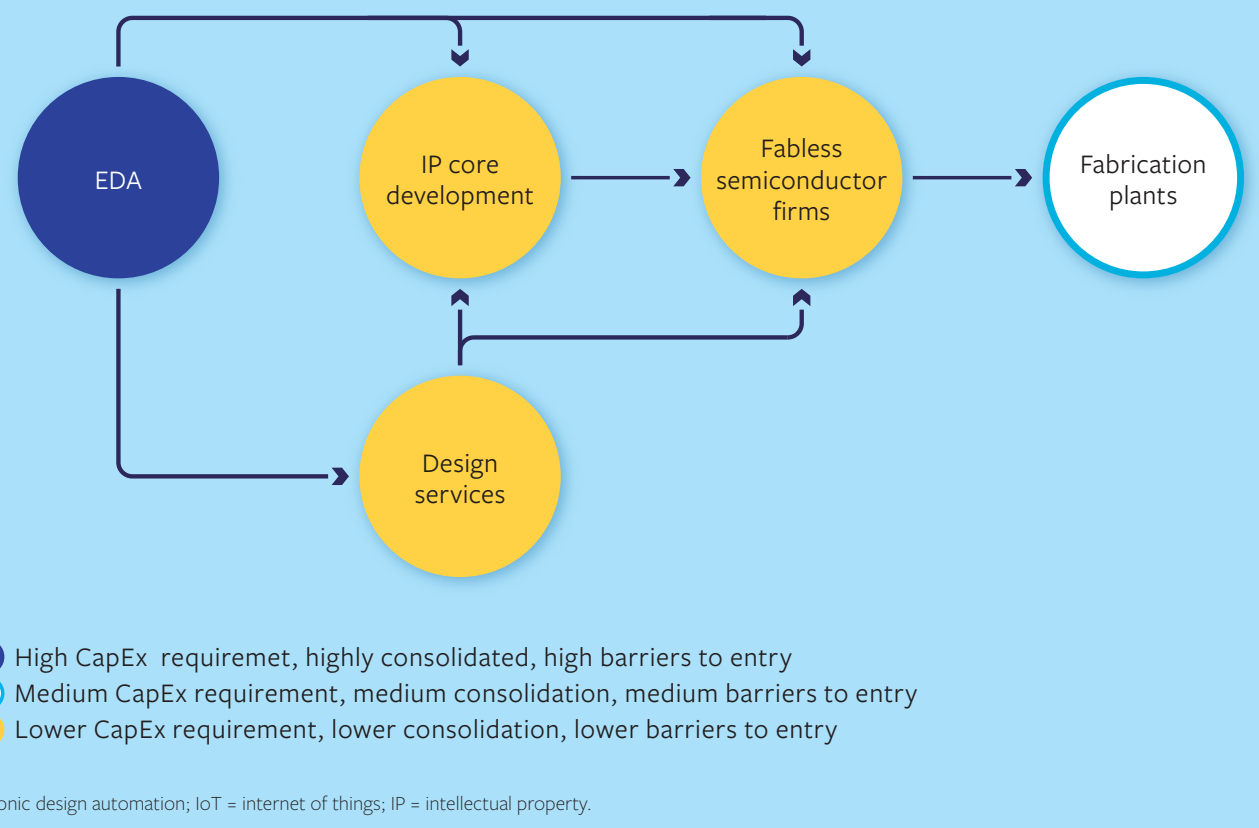

Source: Author's own elaboration.

Note: $\mathrm{Al}$ = artificial intelligence; $\mathrm{EDA}$ = electronic design automation; $\mathrm{lO} \mathrm{T}$ = internet of things; IP = intellectual property 
reduces the ease with which the global semiconductor industry can collaborate with Armenian firms in these cutting-edge technologies. Because EDA is not covered on the lists, this has not been a problem so far. But as Armenia seeks to upgrade participation in semiconductor value chains, the Armenian government may consider working with the U.S. Department of Commerce to address security concerns and improve Armenia's grouping.

2.3

\section{BUILDING CAPACITY AND RELEVANCE IN NASCENT TECHNOLOGIES WHILE THEY ARE STILL ACCESSIBLE}

\section{QUANTUM COMPUTING R\&D}

\section{Quantum computing research is still in its infancy.}

In the early 1900s, a number of theories were put forward to explain peculiar observations in the behavior of subatomic particles. Up until that time, the assumption was that two-state systems, such as the spin of an electron (up or down) or the polarization of a photon (horizontal or vertical), could only exist in one state or the other. However, it was discovered that, at atomic scales and smaller, some systems (called qubits or quantum bits) can exist in both states at the same time. These nowproven theories form the basis of quantum computing; a computer that could harness these (and other) subatomic behaviors would be exponentially faster than existing "classical" computers. The possible implications for the global economy are vast, with applications across the economic spectrum, including finance, energy, pharmaceutical, and logistics. The challenge has been in recreating these behaviors in the physical world in a way that can be controlled and manipulated. There are many possible approaches, but over the past 20 years research has coalesced around five or six. Several prototype quantum computers have now been constructed based on these approaches, but at the time of writing, ${ }^{16}$ no prototype has been able to overcome fundamental noise issues, making quantum computers no more powerful than classical computers. The global quantum research community is therefore yet to demonstrate "quantum supremacy"-proof that quantum computing devices can solve problems that would otherwise be impossible ${ }^{17}$ for classical computers to tackle.

Only a handful of entities globally have had the capital, knowledge, and convening power to construct prototype quantum computers. A small number of technology giants (such as IBM, Google, and Intel) and start-ups backed by venture capital (such as Rigetti, IonQ, and Quantum Circuits) have been able to build prototype quantum computers or hardware subsystems. Although some universities and research institutions may have also built their own prototypes, given the complexity and capital requirements involved in recreating quantum conditions (let alone in a way that is "programmable"), quantum computing prototype construction will likely remain highly consolidated for the time being.

However, outside of hardware development, the quantum research ecosystem is vast, and the global playing field is level. Many of the most prominent firms that have constructed prototype quantum computers also offer free access to their prototypes via the cloud. IBM, Rigetti, Google, Alibaba, Microsoft, and D-Wave all offer such services, allowing users around the world to run algorithms and experiments, and to publish research derived through each platform. In so doing, hardware developers are able to "crowdsource" development and at the same time build an open source community around their solutions as the race toward quantum supremacy continues. Meanwhile, the cloud computing model has meant that physicists, data scientists, and mathematicians have been able to begin to research algorithms for a range of vertical applications. There are also opportunities in "upstream" development, for instance, in core infrastructure redesign, performance enhancement or documentation improvement. As an example, one of the limitations of quantum computers is their susceptibility to changes in their surrounding environment and their consequent tendency to produce errors. Small start-ups and independent researchers are actively engaged in designing software for error mitigation and are attracting significant investment in doing so.

State-sponsored quantum programs historically formed the backbone of quantum computing research. Just as quantum computing promises a range of economic benefits, quantum computing also threatens the encryption systems that are ubiquitous in today's digital infrastructure. ${ }^{18}$ This includes the global finance and monetary systems, digital health systems, and other systems that depend on data protection and secrecy. These concerns have prompted governments to invest heavily in quantum computing. China leads the way, having announced plans to open a 92-acre, US $\$ 10$ billion quantum research center by 2020 . In direct recognition of the data security concerns, the 
United States passed the US\$1.275 billion National Quantum Initiative Act in 2017. The EU similarly launched the Quantum Technologies Flagship in 2018, a 10-year $€ 1$ billion research initiative. Other countries with large state-sponsored programs include Australia, Canada, Israel, Japan, Sweden, and the United Kingdom.

However, private sector funding for quantum research now dominates, opening the door to researchers in middle-and low-income countries. Large multinationals and venture capital funds have dwarfed state-sponsored quantum research in recent years. IBM invested US\$38 billion in a quantum computing program between 2013 and 2018 (Green 2018). But research into quantum computing has also revealed commercially valuable discoveries in other fields (e.g., quantum communication, quantum metrology, and quantum simulation). Private firms that may not have been actively engaged in developing their own quantum solutions are now funding independent research. As a result, research labs in middle- and low-income countries that are unable to benefit from state-sponsored opportunities have much greater prospects of participating in global quantum computing development, armed with little more than a laptop and an internet connection.

Global citations of Armenia's R\&D in physics far exceed those for mathematics and computer science, putting Armenia in the top 25 countries in the world for R\&D citations in nuclear physics. As described previously (see section 2.2), Armenia's teaching institutions have struggled to keep pace with dynamic disciplines such as engineering and computer science. However, global advances in natural sciences, particularly foundational physics and mathematics, have been slow, or even stagnant for 50 years. Despite concerns about integrity in Armenia's tertiary education programs, Armenia's physics curricula and research output remain industry-relevant. This includes PhD-level research and is evidenced by the number of (internationally) cited documents that Armenia's physics community publishes each year (figure 2.9). In 2018, Armenia ranked 25th in the world for the number of citations related to nuclear and high energy physics-key facets of quantum physics. ${ }^{19}$

\section{Armenia's strong background in EDA is also} highly relevant to quantum computing development. EDA software is used to design semiconductors, and Armenia already has a strong foothold in this area (see section 2.2). Several start-ups and research institutions are investigating the use of EDA techniques for fully automatic quantum compilation and programming. Conceptually, both activities seek to map a theoretical computational algorithm to a physical computing device. Some of the most prominent approaches to quantum computing-used by IBM, Google, and Intel-use semiconductors as an integration platform for their fundamental information building blocks (qubits). If Armenia is able to build on its core competence in EDA and move toward semiconductor design itself, there may also be opportunities for crossover and collaborative activities and research.

Nonetheless, Armenia has no national quantum strategy and is not leveraging its potential comparative advantage in this area. There is only one quantum computing lab in Armenia at the time of writing this report, and no formal training at the bachelor's, master's, or doctoral levels. As the global race intensifies, Armenia may need to develop a quantum computing strategy and road map, at the very least for reasons of security, and to stake its position. In so doing, the government could consider policies that encourage the international research collaboration in the field of quantum research, position Armenia globally as a regular "stop" on the quantum computing conference circuit, introduce student sponsorship and exchange programs and, where possible, make targeted investments in quantum computing research. 


\section{FIGURE 2.9}

Citations of Armenian research papers in international journals, 2018

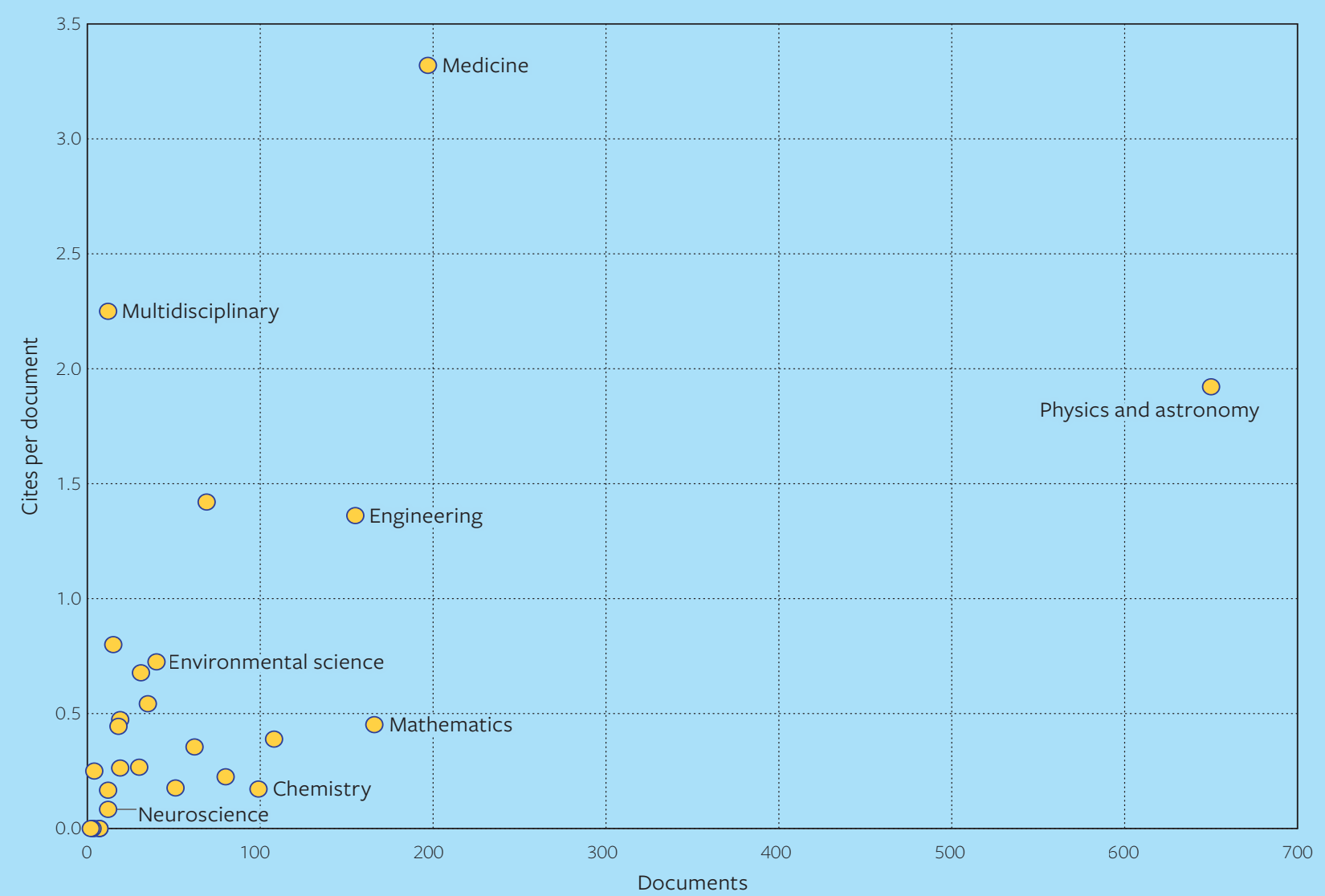

Source: Scimago Institutions Rankings database. 


\section{ICT SERVICES FOR DIGITAL HEALTH AND BIOTECH APPLICATIONS}

\section{Armenia's potential to deliver ICT services for biotech applications should not be constrained by the lack of local demand}

The local market for digital health solutions is underdeveloped in Armenia. Government per capita spending on health care is comparatively low (table CS2.1). Though private spending is quite high, the preventative digital health care solutions market is still in its infancy in Armenia. As a proxy for the reduced market consumer awareness and concern for wellness, consider that 52 percent of Armenian men smoked in 2016 compared with an average of 38.4 percent across Europe and Central Asia. ${ }^{a}$

However, the global market for digital health applications is projected to reach US\$504.4 billion by 2025 . Armenian start-ups are already providing some hospital management solutions to foreign clients, but globally, digital health includes a vast array of markets and applications. Consumer-facing mobile applications have enjoyed a surge in popularity in recent years, focusing on wellness management considerations such as diet, nutrition, lifestyle, and fitness. Other "health condition management" solutions are more clinical (for example, medication management

\section{TABLE CS2.1}

Health care expenditure per capita, 2016

Current US\$PPP

\begin{tabular}{|lrr|} 
& Private & Public \\
\hline Armenia & $\mathbf{2 9 4}$ & $\mathbf{5 9}$ \\
\hline Europe and Central Asia & 467 & 1,625 \\
\hline Central Europe and the Baltics & 239 & 613 \\
\hline Small states & 190 & 376 \\
\hline World & 260 & 763 \\
\hline
\end{tabular}

Source: World Development Indicators database.

Note: PPP = purchasing power parity. 
or women's health and pregnancy solutions). However, digital health development includes many more technologically complex applications in areas in which Armenia is not yet participating. These areas include diagnostics and treatment, disease and epidemic prediction and tracking, and remote monitoring devices and services. In 2018, digital health firms in the United States alone attracted US\$8.1 billion of investment-a 42 percent increase over the previous year (Day and Zweig 2019) — and the global market could reach US $\$ 504.4$ billion by 2025 (Ugalmugale and Swain 2019).

\section{Digital health development is reliant on access to data and strong software development capacity- Armenia has both}

In December 2017, Armenia began nationwide implementation of the zArmenia National Electronic Health Information System (ArMed). In 2012, the government designed and approved a program to introduce an e-health system. After five years of development and a successful pilot in six health care facilities, a National Electronic Health Operator was established in December 2017, and the program began rollout. As of June 2019, the system is already serving 55 percent of the population.

Unlike many other digitized health records, ArMed data are accessible through one centralized database. Several countries have electronic health records (EHR) systems. However, in countries such as the United States, where different health care providers operate different systems, accessing the data can be bureaucratic and complex. Even countries such as the United Kingdom, which has a national health care authority, can have problems; as of April 2017, a case study by the Centre for Public Impact found that many hospitals in the U.K. still lack a comprehensive electronic patient record system (Basu 2017), and different health care trusts may be served by different information technology (IT) suppliers. ArMed is a single nationwide system, accessible through one portal, collating data from 474 organizations, including hospitals, dental clinics, diagnostic centers, and insurance companies.

In addition, ArMed has been deliberately designed to make its data conducive to digital health application development. When sourcing data from several different EHR systems, issues relating to compatibility or comparability can arise. This is especially true when databases are designed with differing emphases (e.g., patient engagement vs. performance measurement vs. reducing costs). In contrast, ArMed was designed not only to improve access and quality of health care but also to facilitate medical education and research.

Start-ups in Armenia's software development community can use ArMed data to design solutions for global markets. Armenia has strong competence in software development and is gradually moving away from an outsourcing model toward original products and solutions. Growing adoption of health care IT solutions globally will increase demand for software solutions for a variety of users, including physicians, insurance companies, research centers and pharmacies. Moreover, as consumer demand for health applications grows, Armenia's software development community can utilize EHR data to research and develop mobile health and wellness applications. Solutions to protect data and the privacy of health care records are another a growing subset of the digital health sector on which Armenian software developers may also be able to capitalize.

\section{Armenia's population has distinctive health characteristics that could be valuable to global biotech development}

A global rise in chronic diseases is fueling growth in the market for remote patient monitoring solutions. Aging populations, urbanization, and the shift from labor economies to knowledge economies has led to a rise in the incidence of chronic illnesses such as diabetes, cardiovascular diseases, and asthma. This increased prevalence is fueling demand for remote patient monitoring solutions. Remote patient monitoring allows patients and clinicians to track medical and health-related information. Estimates differ, but, on average, the global market for remote patient monitoring technologies is expected to rise to about US $\$ 2$ billion by 2023 .

Chronic diseases are unusually prevalent in Armenia. Sadly, Armenia is an outlier with respect to the incidence, prevalence, and mortality rates of chronic noninfectious diseases such as cancer, 
cardiovascular illnesses, and neurodegenerative diseases. Out of 183 countries monitored by the World Health Organization, Armenia has the second-highest cancer mortality rate in the world for both men and women. In men, the age-standardized rate reached 354.5 deaths per 100,000 persons in 2016 (figure CS2.1). ${ }^{\mathrm{c}}$ This is compared with a 2018 average of 83.4 deaths per 100,000 persons in countries with a medium Human Development Index, and a world average of 122.7 deaths per 100,000 persons (Bray et al. 2018).

The design and development of remote patient monitoring biotech is reliant on access to patients with chronic diseases, in addition to raw data. Remote patient monitoring technologies and solutions include implantable devices (e.g., cardioverter defibrillators), wearable devices (e.g., continuous glucose monitors and cardiac monitors), and internet of things solutions such as sensors for smart watches and mobile applications. Patients with chronic diseases are crucial stakeholders in the research and development of remote patient monitoring biotech solutions. In addition to the concentrated pool of data that ArMed is generating.
Armenian patients could participate in clinical trials, provide feedback, and influence the design of these devices globally.

Data related to chronic diseases can also inform the design of preventative health care and telehealth software and hardware solutions. New business models such as preventative health care and telemedicine are starting to gain traction at both ends of the economic development spectrum through their potential to reduce health care costs. Preventative health care business models place greater emphasis on preventative care and wellness than traditional health care business models. In preventative health care, care plans are personalized to the individual, and personalized treatments are delivered at a precise time and place, as appropriate to the patient in question. Telehealth solutions go beyond remote monitoring devices to include (for instance) communication technologies for "real-time" telemedicine (including video and audio medical assessments and basic examinations), as well as diagnostic imaging and interpretation solutions. Both preventative health care solutions and telehealth technology depend

\section{Male cancer mortality rates in 183 countries, 2016}

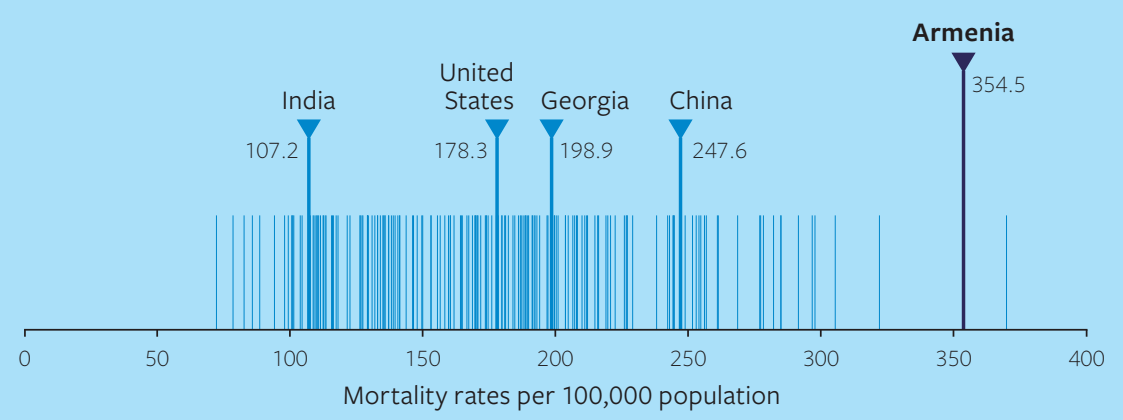

on the growing chronic health community for their experience and insight, as well as for clinical data.

\section{The presumed heterogeneity of the Armenian genome could give Armenia a comparative advantage in the research and development of further applied bioscience solutions}

Genome sequencing has become a core area of applied biosciences. Genome sequencing refers to the large-scale comparison of DNA, sequences of populations. The scale and complexity of genome sequencing projects have meant that many have been publicly funded. National genome sequencing projects enable scientists to characterize the population-level genetic background and investigate the prevalence of various traits and disorders (Arakelyan and Glorikian 2019). This area of genomics has applications in digital health, biotechnology, environmental applications, forensics, and security (Batelle Memorial Institute 2013). Currently, 50 countries around the world have population sequencing programs.

The Armenian genome may be distinct in that Armenia's population is thought to be genetically homogenous. The Armenian government has stated its intention to embark on an Armenian Genome Project (AGP). This could be significant as there is some expectation in the genomic research community that Armenia has a genetically homogenous population. Homogenous genomes are 
easier to use as a basis for research, as heterogeneity can create "noise" making results more difficult to isolate and interactions easier to identify.

If the data from the AGP were to be integrated with Armenia's ArMed platform, the potential could be even greater. Integrating data from the AGP could amplify the potential of both initiatives. Not least, it would enable the comparison of individual patient variants with AGP variants and facilitate outcome monitoring at both the individual and aggregate level. This could help identify novel genetic variants linked to chronic diseases prevalent in Armenia, such as cancer, cardiovascular disease and neurodegeneration (Arakelyan and Glorikian 2019).

The economic impacts of human genome projects in other countries have been significant. Battelle Memorial Institute (2013) has estimated that, between 1988 and 2012, the U.S. federal government's investment of US\$3.8 billion in the Human Genome Project generated economic output of US\$965 billion, US $\$ 462$ billion of value added, personal income exceeding US\$293 billion, and more than 310,000 jobs. ${ }^{\mathrm{d}}$ San Diego, a city of 1.4 million people, is the most patent intensive genomics ecosystem in the United States and received nearly one-quarter of all genomics venture capital in the United States in 2016. An input-output study by BW Research calculated the annual economic impact of its genomics industry to be US\$5.6 billion, supported by 115 core genomics firms employing over 10,000 people (San Diego Regional EDC 2016).

\section{With an appropriate regulatory framework, Armenia can position itself as a global hub for digital health, biotech technology development and bioscience research}

The Armenian government would need to carefully draft localization and data sovereignty laws that define limitations for accessing data from outside Armenia. Many of the opportunities described in this case study are predicated on the assumption that firms must locate themselves in the country in order to access data. However, rather than placing a blanket ban on data access, the Armenian government has the opportunity to draft localization and data sovereignty laws that do not stifle interest but still stimulate local economic activity in Armenia.

The Armenian government and the administrators of ArMed and AGP might consider designing reachthrough licensing agreements and other smart solutions to monetize the data. "Reach-through" agreements allow upstream technology firms to earn royalties from sales or use of downstream solutions based on their technologies. The practice is most common in pharmaceuticals and could have wider application as the discipline merges with other sectors as part of the digital and preventative health movements. The government and the administrators of ArMed and AGP may consider this and other innovative monetization strategies to provide firms with incentives to pay to access Armenia's data resources.
A complementary FDI strategy could further attract global industry stakeholders, making a significant contribution to local capacity development. A targeted investment promotion and attraction strategy could complement the policies described herein. World-class research and medical institutions, pharmaceutical companies, and biotech organizations could make significant contributions to local capacity development (figure CS2.3).

$-$

a. World Development Indicators database.

b. Coherent Market Insights (2017); Sumant and Jaiswal (2016); Grand View Research (2019).

c. Jobs number does not include 2012 figures.

d. World Health Organization's Global Health Observatory data repository, http://apps.who.int/gho/data/view.main. A1110. 


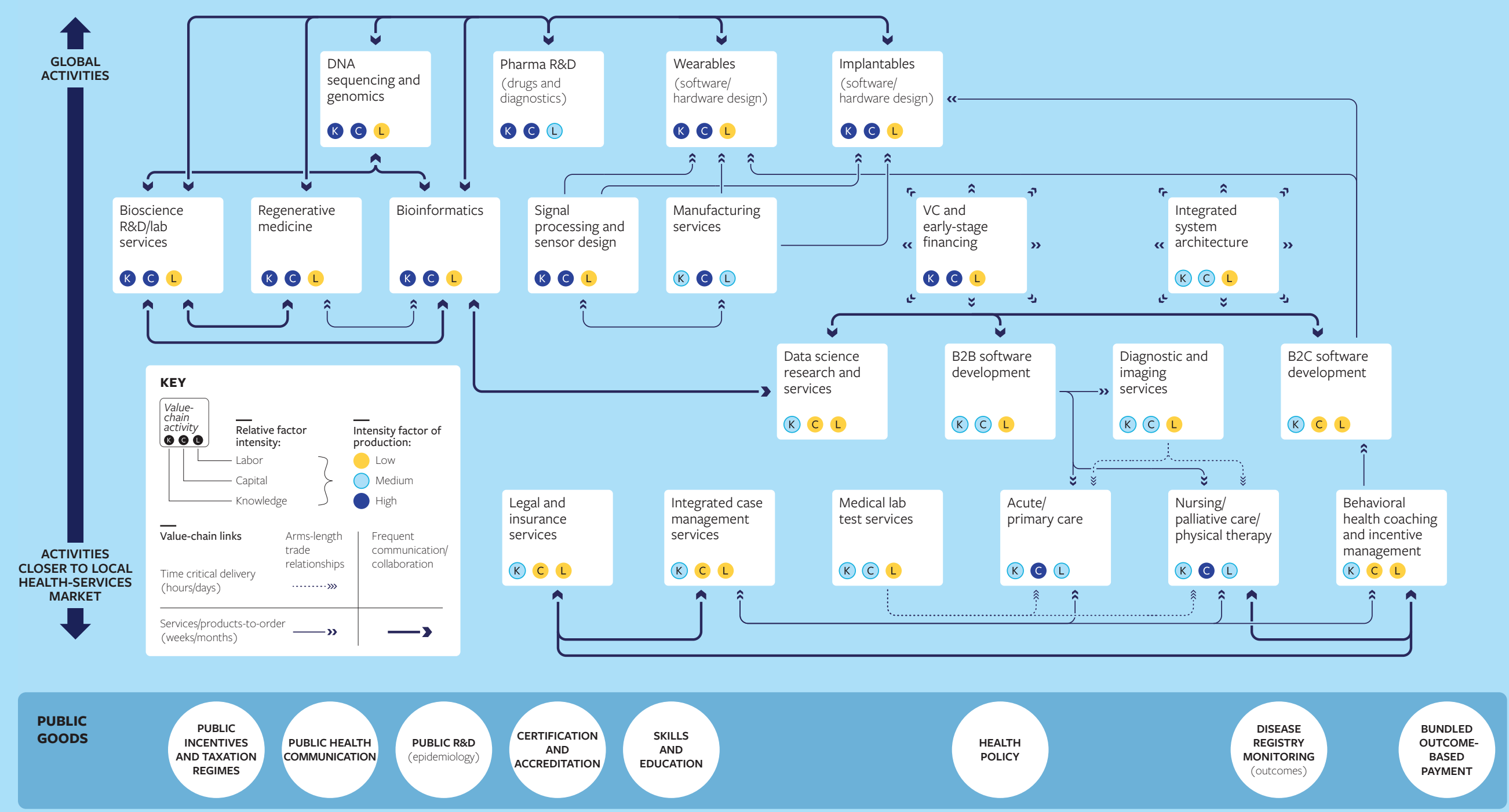


FIGURE CS2.3

Armenia's participation in the biotech/digital health value chain

$\langle$ UPSTREAM
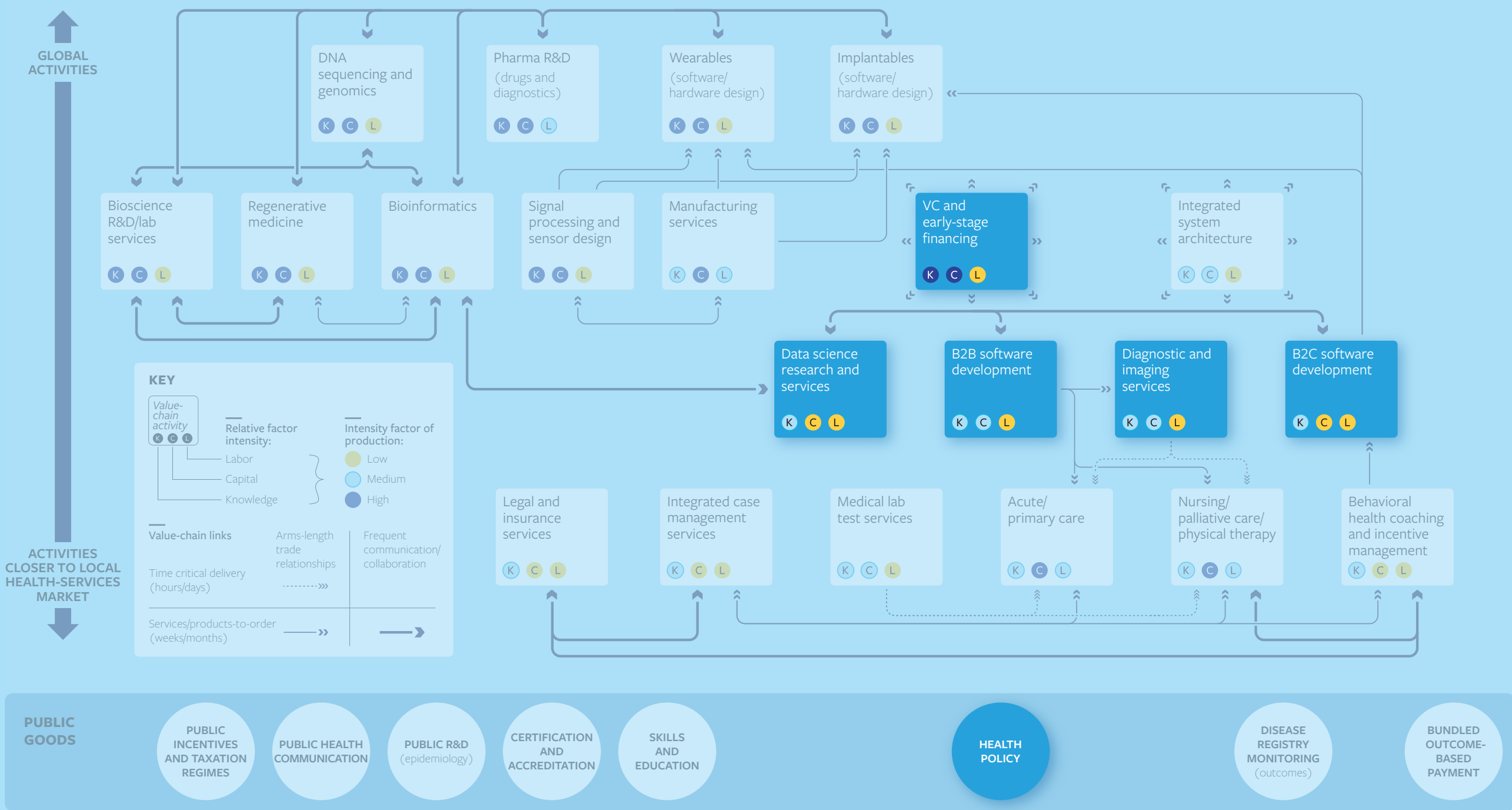

Source: Author's own elaboration

participation in the biotech/digital health value chain. Armenia is already participating in downstream software development activities (highlighted in yellow) but is yet to participate in higher-value-added upstream activities including bioscience R\&D and lab services, bioinformatics, wearables or signal processing. Close-to-market, higher-value-added activities like "integrated case management" require presence in target market and local expertise. 


\section{Chapter 3}

\section{STRENGTHENING THE ECOSYSTEM}

\begin{abstract}
A stronger regulatory environment is key if Armenian firms are to successfully compete for higher-value-added activities. Structural weaknesses in Armenia's regulatory ecosystem are constraining the potential for start-ups and young firms to participate more in high-tech global value chains (GVCs). First and foremost, funding gaps and legal restrictions have made local incorporation unattractive. At the same time, legislation governing foreign direct investment (FDI) is somewhat outdated, limiting inward investment. As a result, Armenian firms in high-tech sectors have incentives to incorporate abroad even when their operations are to remain in Armenia, limiting local tax revenue and spillovers. Further upstream, Armenia's educational institutions are not adequately preparing students. These themes are discussed in the following sections.
\end{abstract}

3.1

\section{INNOVATION AND ENTREPRENEURSHIP}

There is only limited venture capital available in Armenia to support business innovation and entrepreneurial endeavors. Armenia has recently experienced a wave of early stage financing schemes. It started in 2006 with the Enterprise Incubator Foundation's (EIF) Science and Technology Entrepreneurship Program and it continue to imple- ment several financing programs and grant schemes. Since then, other funds have emerged to support Armenia's start-up ecosystem. Armenia's first venture capital fund, Granatus Ventures, was launched in 2013, and the second one, HIVE Ventures, was launched in 2014. In more recent years, several other schemes and platforms have emerged, including SmartGate Venture Capital. Although new funds are in discussion, ${ }^{20}$ Armenia's venture capital envelope is estimated at US $\$ 15$ million (as of July 2019). With little publicly available information and sustained inconsistencies in the reported value of each of these funds (during presentations, events, consultations, and interviews), their value may be much lower.

At the same time, FDI opportunities are constrained by a 1994 law that does not adequately address current investment realities and limits arbitration to Armenian courts. Protection of foreign investments is governed by the law on Foreign Investments of 1994 (or on a country-by-country basis through bilateral treaties). Drafted 25 years ago, the law does not adequately address the current international investment landscape. A revised law could offer more predictable, accessible, and effective protection mechanisms for investors (e.g., defining preconditions for resorting to expropriation or defining characteristics of compensation payment). Investor "aftercare" could be improved by introducing clear mechanisms for 


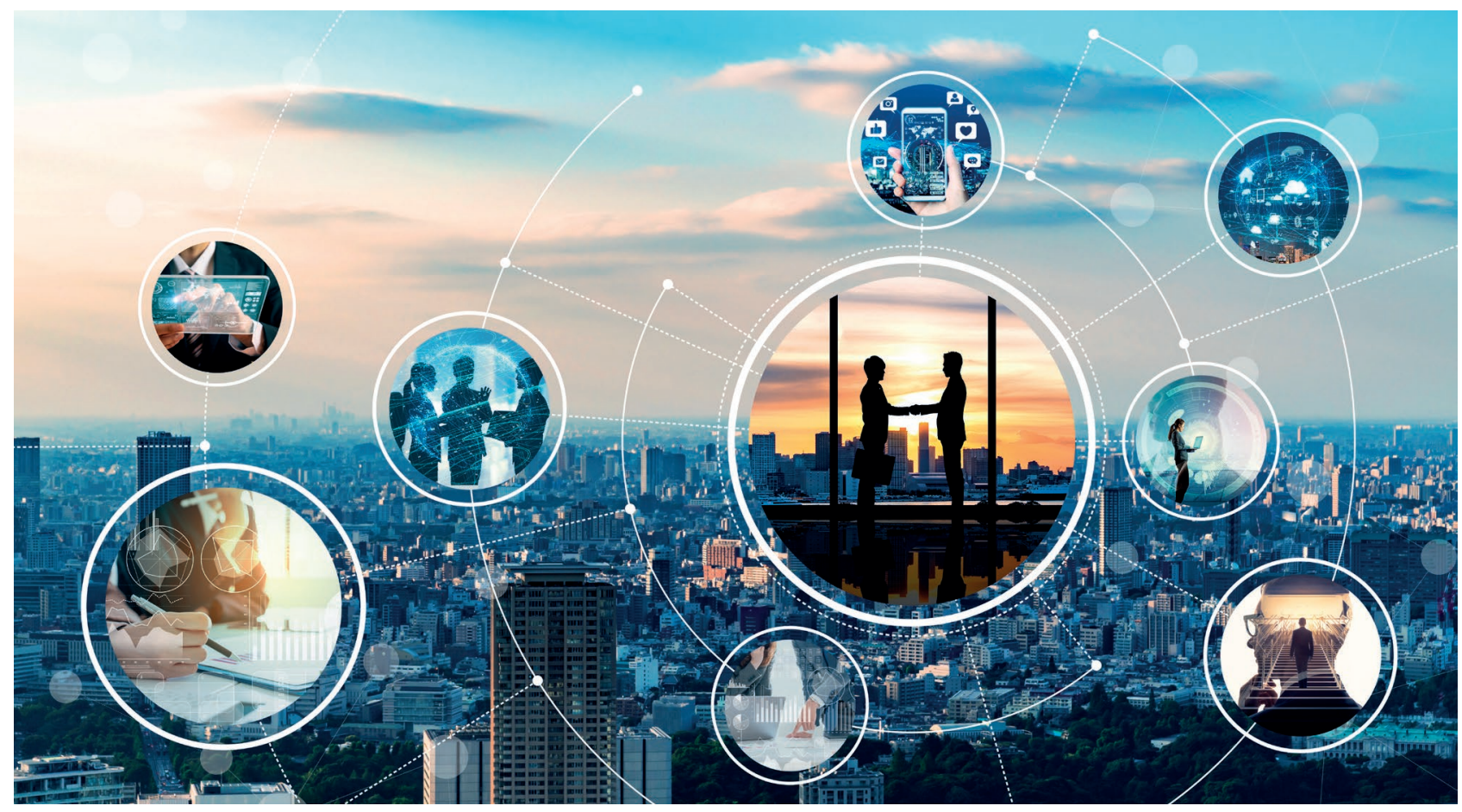

management of grievances and complaints. Crucially, changes to the law could allow investors to use international foreign courts rather than Armenian courts in the case of arbitration, if they prefer.

As a result of these limitations, Armenian firms often incorporate abroad to attract investment, limiting local spillover effects. The lack of early stage financing in Armenia has driven several Armenian tech firms to seek investment abroad. In most cases, investors explicitly request investees to incorporate under laws that the investors are familiar with. This is especially true of U.S. investors. Given that many Armenian firms have grown through U.S. investments (due in large part to strong diaspora links), most Armenian tech start-ups are incorporated in the United States, even if their U.S. headquarters are virtual or only nominally staffed. This firm structure reduces the Armenian entity to subsidiary status, meaning that any investment they receive remains in the U.S. account and U.S. dollar transfers are limited to operational costs, salaries, and overheads. As an example, PicsArt (valued at an estimated US\$250 million) was able to raise capital through two rounds of venture capital funding by incorporating in the United States.

Shortcomings in Armenia's deal flow management and seed-funding schemes are also constraining Armenia's high-tech start-up growth. Many Armenian start-ups are too small to attract venture capital (World Bank 2019). As a result, several initiatives for angel and seed-stage funding have started, including the Business Angel Network of Armenia, the Fast Foundation's Science and Technology Angel Network, and the Angel Investor Club of Armenia, among others. The aforementioned EIF is one of the largest technology business incubators in Armenia, which aims to create a productive environment for innovation, start-up creation, and higher productivity. In addition, several other incubators, accelerators, coworking spaces, business centers, and entrepreneur associations support start-up growth in Armenia. However, thus far, the measurement of success for many of these schemes focuses on the number of startups and entrepreneurs that participate in the scheme rather than follow-on funding, sales, or business viability (of the scheme). As a result, there is very little idea validation across the ecosystem. In Silicon Valley in the United States and other global tech hubs, start-ups with poor market viability would not usually be in an incubator for more than four to six months, because the incubator framework recognizes that the start-up is nonviable and parts ways with entrepreneurs. Conversely, industry stakeholders in Armenia have repeatedly raised concerns during consultations that nonviable start-ups circulate through the ecosystem's incubator and accelerator programs over and over, compromising the efficacy of the ecosystem. As the Armenian govern- 
ment, together with the development community, continues to expand these programs, more emphasis can be placed on managers, trainers, and mentors (in addition to the scheme's monitoring and evaluation structure) to bolster the efficacy of the programs.

Some aspects of Armenia's corporate law are poorly suited to early stage financing and investment. In reflection of their added risk, venture capital and angel investors often expect to acquire preferred stock (preference shares) with liquidation and dividend preference. Early stage investors may also demand some voting power (even if subordinate). Armenian law only allows joint-stock companies to issue preferential stock and must be "nonvoting" shares. There is no legal structure that permits different classes of voting shares, which entail differing entitlements to dividends and different levels of voting power (IP/IT Committee for Armenia 2019). Additionally, where seed funding is received as a debt, converting that investment into equity during subsequent funding rounds (as is common with convertible loans) is not possible under Armenian law.

Finally, intellectual property is not well protected. Attitudes to patenting software differ from industry to industry. Many industries rely on interoperability and open source code, making patenting redundant. Other industries rely on alternative areas of their business to create and retain value (e.g., brand recognition, language and culture, service delivery). However, outside of mass-market applications (e.g., mobile apps, games, health management software, etc.) software patenting is more common. A software that is unassociated with a hardware-based application has traditionally been difficult to defend in a court of law, but a software showing "further technical effect" (i.e., one that is integrated with hardware) is more defensible. Armenia has neither legislation to protect "standalone" software nor software that is integrated with hardware.

\section{2}

\section{ACCESS TO GROWTH FINANCE}

\section{Small firms in Armenia rely heavily on internal} resources and retained earnings for growth finance. In 2013, the main source of financing for micro, small, and medium enterprises (MSMEs) in Armenia were the owner's own funds and company resources (World Bank 2013). Friends and relatives were other major sources of financing. Only 55 percent of firms reported financing from commercial banks and only 15 percent sought funds from credit organizations. Put differently, almost three-quarters of firms were financing their investments internally in fixed assets and working capital rather than through banks.

Promotion of financial technology innovations (e.g., mobile banking) and use of agents could help expand outreach to underserved segments and locations. The government can develop a fintech strategy to bridge financing gaps for small firms and expand outreach at reduced costs. In doing so, leading finance institutions can better address information asymmetries and reduce risks in lending, facilitate market monitoring, and introduce innovative products. With adequate regulatory safeguards and oversight, agents (e.g., the Armenian Post) could support delivery of core financial services through partnership or agency agreements with financial institutions.

\section{At the same time, small firms need additional} business and financial management training to take advantage of such schemes. Low levels of product awareness and financial management capacity in the MSME sector are considerably affecting firms' ability to select adequate financial instruments. Additionally, business, financial, and strategic planning are rare. In general, investment plans are frequently developed without a broader long-term vision for the enterprise or a solid assessment of the viability, risk, or expected cash flow of the investment. As a result, financial institutions report that smaller enterprises often apply for loans they cannot afford and face significant challenges in repayment.

There is already a high level of informality in the enterprise sector, which may be exacerbated in software development activities. A 2018 International Monetary Fund study (Medina and Schneider 2018) estimates Armenia's informal economy at around 36 percent of its overall gross domestic product (GDP) in 2015, slowly declining below 40 percent since 2011. Although Armenia's shadow (i.e., informal) economy is much smaller than other countries in the region (Georgia's is 53 percent while Ukraine's is 43 percent), it is significantly larger than in other comparable countries such as Bosnia and Herzegovina (30 percent), Albania (26 percent), or Croatia (23 percent) and even double the size of those in Estonia and the Islamic Republic of Iran (18 percent). Software development activities, where barriers to entry are low, are susceptible to informality. In addition, cash-on-delivery is the most popular payment method in the nascent e-com- 
merce market. (As an example, only 9 percent of credit card payments are attributed to online transactions). Pervasive informality will affect the potential of firms to access to credit, because lenders will not be able to use cash flow-based credit analysis to assess viability.

\section{3}

\section{DIGITAL TRADE}

\section{Armenian law recognizes e-documents and digital} signatures but could go further to allow contracting parties to select the technology that best suits their needs. A conducive regulatory framework for digital trade should guarantee that contracts concluded remotely through electronic channels are valid and legally enforceable just as those concluded in person. Armenia's Law on Electronic Document and Electronic Signature and the Civil Code recognize electronic documents as equivalent to paper-based documents. Additionally, the law is technology-neutral regarding storage of e-documents (allowing parties to decide what is most appropriate based on their circumstances). However, the law only recognizes digital signatures ${ }^{22}$ as legally valid rather than allowing contracting parties to select the technology that best suits their needs. The Armenian government could consider expanding the law to recognize all electronic signatures and could, in addition, grant them "presumption of validity and authenticity" in legal proceedings.

Cybersecurity provision could enhance Armenia's already strong framework for online data protection. In 2015, Armenia introduced a comprehensive legislation to protect online personal data privacy, taking elements from the European Union Data Protection Directive of 1995. The Law on Personal Data Protection includes important provisions such as special rules for the treatment of sensitive personal data, legal bases of data collection and processing, data subjects' rights to access and deletion, and rules on cross-border data transfers. However, although the law includes a framework for cybersecurity with requirements such as data encryption and breach notification, it lacks certain recommended provisions. The Agency for the Protection of Personal Data could consider including specific regulations for cybersecurity, adding requirements such as the appointment of a data manager and risk assessment procedures.

The Armenian government does not yet have a framework that addresses intermediary liability.
Intermediary liability regulations distribute liability between intermediaries (e.g., websites and apps) and online vendors or content developers in the event of a dispute. Rules on intermediary liability need to strike a balance between protecting consumer rights and supporting the expansion of digital markets, including through intermediary platforms. Digital intermediaries manage the relationship with the customer, and they are often the largest, most sophisticated actors involved in a transaction. As such, regulations can impose intermediary liability for fake or faulty products or services, or for offensive or illegal content transacted through their services. Armenia lacks a specific framework to address online intermediary liability. The government could consider a framework that explicitly addresses online intermediaries, with safe harbor provisions to shield them from liability for infringing content posted by third parties, including intellectual property violations.

3.4

\section{RESEARCH AND EDUCATION}

\section{The Armenian government's support for research} and development (R\&D) has largely focused on infrastructure investments. Public funding for Armenia's science, technology, and research potential have thus far been focused on infrastructure (e.g., technology parks, centers of excellence, labs). Examples include the Gyumri Technology Centre and the Vanadzor Technology Centre. More recently, "Engineering City" in Yerevan is under construction, aiming to create an environment for engineering firms to accelerate the development of complex engineering solutions. Facilities will include research and prototyping labs, machine tooling, and advanced production equipment.

There is an opportunity to prioritize research areas and implement targeted $R \& D$ policies. Sections 2.2 and 2.3 detail Armenia's potential in pure and applied research in fields related to natural sciences and mathematics. Notably, Armenia has high potential in the areas of AI pure research and quantum computing R\&D. Armenia's potential in high-tech sectors is closely tied with its R\&D framework. An increase in public funding for R\&D would also be necessary to realize Armenia's potential; the country spent 0.2 percent of its GDP on R\&D in 2017 compared with a world average of 2.2 percent. Additional budget could fund targeted research policies including applied 


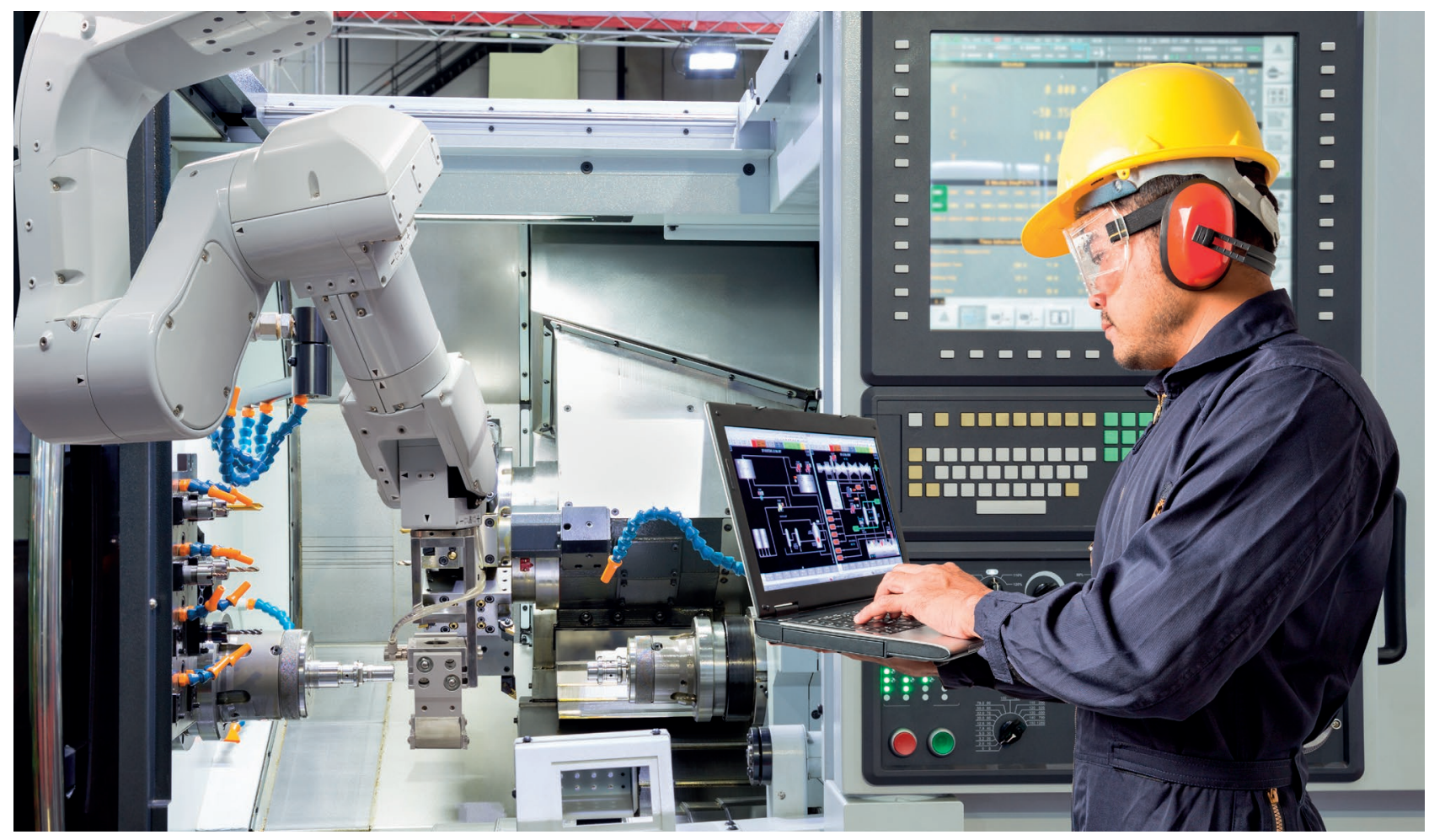

research grants, competitive research collaborations, competitions and R\&D tax credits. There are plans to develop 24 separate sectoral strategies including a High-Tech Industry Strategy (under the Ministry of High-Tech Sectors) that will be separate from the Education, Science and Research Strategy (under the Ministry of Education, Science, Culture and Sports). The Armenian government should go beyond developing "complementary" strategies and rather intertwine the two strategies.

\section{Armenia's engineering and computer science} curricula need improvement and regular review. Armenia's engineering and computer science courses are severely lagging industry. Engineering courses are particularly out of date, with few courses incorporating computer-aided design software (e.g., Autocad or Solidworks) or printed circuit board software (e.g., Altium or Orcad). Although computer science disciplines have seen some improvement in recent years, graduates are still not industry-ready or even "industry-relevant" in some cases. As an example, prominent universities such as the National Polytechnic University of Armenia (NPUA) are still teaching Turbo Pascal, a programming language developed in the 1960s that was superseded by C, C++, and Java in the 1990s. Individual universities are also taking the lead themselves; e.g., NPUA's partnership with Rutgers University, National Instruments and EIF to offer a master's program in
Systems Engineering; the establishment of the Armenian National Engineering Laboratories at the NPUA which upgraded outdated laboratory equipment and modernized the engineering curriculum; and the Armenian-Indian Center for Excellence in Information and Communication Technologies established in Yerevan state University. However, as the World Development Report 2020: Trading for Development in the Age of Global Value Chains notes, in lieu of public sector progress, employer-sponsored training within GVCs can become a mechanism for skills development. This is clearly evident in the case of Synopsys, which operates its own bachelor's, master's, and research programs at its educational center and at the NPUA, Yerevan State University, the Russian-Armenian (Slavonic) University, the European Regional Academy and the National Research University of Electronic Technology. Not only are Armenia's engineering and computer science courses in need of overhaul, public universities can better work with the private sector to provide more professional development and on-the-job training options that provide mid-career learning options. Providing training through nimble and dynamic learning tools-such as certificate programs, single classes, and massive open online courses-will help universities to adapt quickly to changing and emerging technologies. 


\section{Chapter 4}

\section{ACTIONS AND RECOMMENDATIONS}

This chapter introduces recommendations that could strengthen Armenia's participation in hightech value chains through policy reforms, capital investments, and skills development (table 4.1).

As the discussion in the previous chapters has demonstrated, any strategic activity to reposition Armenia in high-tech value chains will require strong administrative and coordination capacity in government. In particular, the Ministry of HighTech Industries and the Ministry of Education, Science, Culture and Sports need a joint operating framework that will allow them to codesign and co-implement an overarching innovation strategy for Armenia.

It is worth noting also that a simple change in government policy may not be enough; the Armenian government may need to employ change management approaches to maximize the impact of reforms. As an example, public-private dialogue mechanisms could raise awareness and understanding of the reforms and their implications. The government must also make sure to allocate adequate resources to enforcing changes in the law where appropriate (e.g., changes in corporate law) so as not to undermine the reform effort. 


\section{Entrepreneurship, research, and innovation}

》Develop an innovation policy road map that identifies policies that encourage international research collaboration in priority fields.
MoESCS, MoHTI Long term

MoESCS,
MoHTI

Provide support for research and innovation programs through subfinancing, e.g., applied research grants, competitive research collaborations and matching grants programs for start-ups.

》Strengthen human resources, research excellence, and research commercialization through government-facilitated collaborative research programs with international universities, private sector, and a targeted visa program.

>Develop capacity of technology transfer offices to convert early stage research into market-ready technology and complete deals.

$\begin{array}{ll}\text { MoESCS, } & \text { Long term } \\ \text { MoHTI } & \end{array}$
$\mathrm{MOHTI}$

MoESCS Short term

>Support development of local and diaspora-led business angel networks in Armenia.

> Shift focus of publicly funded seed-funding programs (including incubators and accelerators) toward "successful exits" rather than "quantity of firms" in the program.

> Introduce software patenting legislation to protect software that demonstrates "further technical effect" (in line with EU guidelines).

》 Introduce managerial training programs to build middlemanagement capacity.

\section{Access to growth finance}

》 Launch consultations and develop a fintech strategy to MoF, CBA

Short term introduce innovative products that could help bridge the MSME financing gap.

》Develop implementation strategy (including adequate regulatory safeguards and oversight) to use agents (e.g., the Armenian Post) for delivery of core financial services. MSMEs on financial products, financial reporting, financial management, business planning, and marketing to enhance their bankability and discourage informality. 


\section{Corporate law and foreign direct investment}

》 Introduce debt instruments (e.g., convertible loans) that can be SRLE, MOE, MOF Short term converted to equity in subsequent funding rounds.

》 Introduce corporate entities that support different classes of voting shares (with differing entitlements to dividends and/or different levels of voting power) in order to better reflect early stage financing risks.

\D Develop clear rules pertaining to the fiduciary responsibilities of corporate management teams toward minority shareholders (so that the latter are adequately protected-particularly where the interests of minority shareholders directly clash with those of majority shareholders).

》Introduce legislation that allows investors to choose between local and international courts in the event of arbitration.

》 Strengthen investment "after care" by creating clear mechanisms to identify, track, and manage grievances between investors and public agencies before they escalate into disputes or withdrawals.

》 Repeal the 1994 Foreign Investment Law and enact new umbrella framework for investment in line with international best practice, including:

>Defining scope of application of the "fair and equitable treatment" principle,

>Defining characteristics of compensation payment,

>Defining limits of prohibition of direct and indirect expropriation, and

>Proscribing the rights of the investor to dispose of their profits and export their funds.

》 Build “investor roadmap" to identify permits, processes, and administrative changes that are constraining investments.

> Design investment promotion strategy to attract global firms in niche sectors that can leverage Armenia's particular factor conditions (e.g., biotech and cleantech). 


\section{Digital trade}

》 Expand the Law on Electronic Document and Electronic Signature to recognize all electronic signatures and grant MoJ Long term them, in addition, "presumption of validity and authenticity" in legal proceedings.

》 Include specific regulations for cybersecurity in the Law on Personal Data Protection, adding requirements such as the appointment of a data manager and risk assessment procedures.

Develop a framework that explicitly addresses online intermediaries with safe harbor provisions to shield them from liability for infringing content posted by third parties, including intellectual property violation.

》) Work with the U.S. Department of Commerce and international counterparts to improve Armenia's grouping in order to facilitate trade and collaboration in "dual-use" technologies as per the international Wassenaar Agreement.

\section{Data policy}

》 Implement policies relating to the classification, formatting, and storage of administrative and statistical data.

》 Implement policies that will enhance digital data management capacity and promote digitization among private firms.

》 Implement localization and data sovereignty laws that do not stifle interest but still stimulate local economic activity in Armenia.

Provide technical assistance to help the private sector comply with the EU's General Data Protection Regulations and meet international standards (e.g., the International Organization for Standardization-International Electrotechnical Commission 27000 family of standards).

\section{Education}

》 Integrate the planned High-Tech Industries Strategy with the MoESCS, MoHTI Short term planned Education, Science, Culture, and Sports Strategy.

> Update bachelor's, master's, and PhD curricula in the fields of computer science and engineering to correspond with industry standards. 
》Introduce annual curriculum review process that uses publicMoESCS, MoHTI Short term private dialogue to solicit industry opinion and incorporates clear mechanism for revisions and updates.

》 Build an artificial intelligence PhD program and identify MoESCS, MoHTI Short term opportunities for joint research labs in collaboration with leading international research institutions (in both the public and private sectors) in this field.

》Develop a quantum computing strategy and road map. This should include formal tertiary training in quantum computing at all three levels-bachelor's, master's, and doctoral.

» Strengthen future incarnations of the Programme for AntiCorruption Measures in Education as follows:

> Provide adequate funding for coordination, monitoring, and implementation of all listed anti-corruption measures,

> Reduce prevalence of short-term, noncompetitive appointments to increase employment security and predictability among university staff,

$>$ Introduce compliance and integrity into accreditation and reaccreditation criteria for higher education providers, and

> Develop comprehensive corruption detection and reporting mechanisms in the higher education sector, in such a way as to protect whistleblowers. 
APPENDIXES 


\section{Appendix A}

\section{APPROACH}

Opportunities for better international integration and insertion into global value chains (GVCs) in various industries depend on a wide array of factors, including global demand trends, sourcing and localization, the strategies of multinational corporations, specific competitive dynamics within each phase and strategic segment of the chain, and the type and rate of innovation within the industry. Some of the factors at least partially under a country's control are the alignment of resource endowments and the provision of complementary public goods, with the required cost structures and minimum efficient scale to compete in a specific phase nationally, regionally, or globally.

Rodrik (2013) has observed recurring patterns of structural change, particularly in East Asia and other high-income countries in recent history. Economies have moved from agriculture and informal economic activities where value addition is low to light manufacturing (e.g., apparel, footwear, simple electronics), then to heavy industries (e.g., automotive, machine tools), and finally to services (e.g., finance, insurance) where productivity and value addition are found to be significantly higher.

Policy makers have accordingly followed that linear logic to foster economic development. However, Rodrik (2014) asserts that globalization and the division of labor, global demand patterns, and the growing emphasis on technology and skills have radically altered that linear development path. Furthermore, the scattered nature of value-chain activities means that the capacity to capture value from a specific industry is determined by the policies of foreign competitors as well as domestic strategy.

Not all value-chain activities can act as stepping-stones to the next. Indeed, each value-chain activity has different potential to catalyze economic development and productive transformation. Therefore, as we move into a new, more uncertain phase of industrialization, it is important to understand where and how value is created within the GVC so as to know which value-chain segments and activities to compete for.

In response, the World Bank Group has developed a qualitative analytical approach to value-chain competitiveness analysis that can better inform project design and help Bank Group clients identify strategic options to retain a greater share of value added from the global industries that they participate in.

This qualitative analysis goes beyond quantifying "how much" value is created by each country participating in a GVC, and instead focuses on how value is created and by whom. It is based on concepts developed by Professor Michael E. Porter and implemented by the European Foundation for Cluster Excellence (in association with the European Union and the Instituto de Estudios Superiores de la Empresa Business School) in Europe and Latin America over the past 15 years. In incorporating this approach in its analytics, the World Bank Group is able to help clients leverage industry-specific public policy and investment options to catalyze private sector development and foster the income growth of the 40 percent of the population with the lowest incomes. 


\section{Appendix B}

\section{METHODOLOGY}

\section{STEP 1}

\section{GLOBAL INDUSTRY DEEP DIVE}

Background and contextual industry deep dive, including industry dynamics and trends related to global supply (historical reason for success in certain countries or regions, ways in which markets are responding to changes in demand), evolution in demand (geography and product or service), industry cost structure and margins, recent evolution in value-chain structure (e.g., localization, specialization, and integration among firms). This work relies mostly on secondary research and secondary data.

\section{STEP 2}

\section{STRATEGIC SEGMENTATION}

Industry "segmentation" that groups each industry not only by product or service but also by intended market or user group using the global industry deep dive in step 1, and Professor Michael E. Porter's "five forces" analytical tool, which was also used to assess industry attractiveness by determining the profitability of the industry and identifying the actors within the industry with the most bargaining power (thereby determining which actors capture the bulk of the available profits). In addition, the analysis included some assessment of the evolution (historical and future) of this attractiveness. In addition to traditional secondary resources (e.g., journals and papers), strategic segmentation relied on interviews with industry experts and nontraditional sources of information such as media (e.g., online newspapers, magazines), annual reports, filings, and blog posts.

\section{STEP 3 \\ IDENTIFICATION OF PROTOTYPICAL VALUE CHAIN}

For each strategic segment identified in step 2, and using similar resources:

a. An Advanced Buyer Purchase Criteria Assessment focused on the minimum requirements (quality, volume, price, safety, transparency, traceability, financial viability) that commercial buyers (both business-to-business and business-to-consumers) would accept.

b. An assessment of geographic strategic options identified the advantages and disadvantages of targeting local or global market using a single product or brand offering or strategy.

c. Key Success Factors-the characteristics that a firm must have to compete well-were identified for each strategic segment.

d. Resources a, b, and c were used to "map" the ideal value chain for each strategic segment and identify:

i. The optimal distribution of activities between the local, regional and global levels of the value chain in terms of minimum efficient scale of operation and production runs;

ii. The type (knowledge-intensive, capital-intensive, labor-intensive, natural resources, and energy-intensive) and level of intensity (low, medium, high) of each value-chain activity; and

iii. Typology of value-chain linkages (just-intime, made-to-order, on-stock) by time ( 24 to 48 hours, weeks, months) and information exchange (high or low information exchange) that are required to compete effectively in a specific strategic segment. 


\section{STEP 4}

\section{ARMENIA'S CURRENT POSITIONING}

A World Bank specialist traveled to Armenia and the United States to identify segments in which Armenia's industries are currently participating. Consultations included almost 50 private firms and institutions in Yerevan, San Diego, Silicon Valley, and Washington D.C. Armenia's performance in identified segments was assessed (compared to regional, as well as global, competitors) and the "ideal value chain" for those segments was compared with the Armenian context. Specific policy, investments and/or institutional interventions that might be required for Armenia to better compete in those segments and capture more value were identified.

\section{STEP 5}

\section{BENCHMARKING AND FEASIBLE}

\section{STRATEGIC OPTIONS}

Strategic repositioning options for Armenia, including higher-value-added segment(s) that could be targeted; viable policy options to maximize value addition within the segment(s); and necessary investments and institutional changes that would be needed to support the development of Armenia's productive capacity within the segment(s) were identified. Washington, D.C.: World Bank Group. 
1. More information on the methodology and the rationale for its selection is included in the appendix.

2. Virtually all the firms that were interviewed manufacture educational electronics toolkits spun out of National Instruments after it had received specific requests for education solutions that were outside of its core business.

3. Homogenous genomes are easier to use as a basis for research because "noise" arising from diverse ethnicities is greatly reduced, making results easier to isolate and interactions easier to identify.

4. Economists play an important role in cost-benefit analysis modelling.

5. Natural or "pure" sciences use quantifiable, empirical research methods to investigate natural phenomena.

6. Assuming (a) the distribution of bachelor's degree programs in 2022 corresponds to the distribution of bachelor's degree enrollments by program in 2018, (b) the ratio of tertiary to bachelor's degree graduates remains consistent, and (c) future tertiary graduate figures are a function of current primary and secondary enrollment figures.

7. Yerevan State University's master's degree program in data science for business is implemented jointly with the Innovative Solutions and Technologies Center and the Enterprise Incubator Foundation. Collaboration with the California State University of San Jose allows professors and researchers to integrate with their U.S. counterparts and thereby better the research institute.

8. Integrated circuits are typically embedded on a thin wafer (or "chip”) of semiconductor materialmost often silicon. This is why they are interchangeably referred to as silicon chips, semiconductor chips, "semiconductors," or “chips."

9. NASDAQ market capitalization on June 21, 2019.

10. Ibid.

11. Mentor Graphics was acquired by Siemens in March 2017 for US\$4.5 billion.

12. NASDAQ market capitalization on July 7, 2019.
13. IP stands for intellectual property and, in this case, is a throwback term to the earliest years of the semiconductor industry.

14. In the semiconductor industry, "process nodes" or simply "nodes" are predefined technology development milestones.

15. Only five countries; Cuba, the Islamic Republic of Iran, the Democratic People's Republic of Korea, Sudan, and the Syrian Arab Republic are in Group E. There are 49 Group D countries; examples include Afghanistan, Belarus, Central African Republic, the Russian Federation, and Zimbabwe.

16. June 24, 2019.

17. Or impractical for a classical computer to solve as it will take thousands of years to finish.

18. Modern encryption techniques rely heavily on asymmetric encryption algorithms that would take centuries (or longer) to break using classical computing. However, in 1994, Peter Shor, an American mathematician, wrote a quantum computer algorithm that could solve encryption algorithms at rates that are exponentially faster. If the necessary quantum hardware were available, it would render current (asymmetric) encryption algorithms ineffective.

19. Scimago Institutions Rankings database.

20. In April 2019, the United Nations Development Programme signed an agreement with Granatus Ventures to help establish and manage the Granatus Tech4SDG Fund, aiming to raise US\$40 million.

21. According to the European Patent Office's guidelines, "further technical effect" is going beyond the "normal" physical interactions between the program (software) and the computer (hardware) on which it runs.

22. "Digital signatures" fall under specific standards and stringent verification process. They explicitly involve the use of a code or algorithm to sign and validate the authenticity of a document. 
ACN (Anti-Corruption Network for Eastern Europe and Central Asia). 2018. Anti-Corruption Reforms in Armenia: 4th Round of Monitoring of the Istanbul Anti-Corruption Action Plan. Paris: Organisation for Economic Co-operation and Development. https:// www.oecd.org/corruption/acn/OECD-ACN-Armenia-4th-Round-Monitoring-Report-July-2018-ENG.pdf.

Altbach, Philip G., Liz Reisberg, Maria Yudkevich, Gregory Androushchak, and Iván F. Pacheco. 2012. Paying the Professoriate. Routledge. https://www. bc.edu/content/dam/files/research_sites/cihe/pdf/ IHEpdfs/ihe68.pdf.

Arakelyan, Arsen, and Harry Glorikian. 2019. "The Armenian Genome Project Implmentation Strategy \& Roadmap."

Basu, Diptasri. 2017. "The Electronic Health Records System In the UK." Centre for Public Impact, London. https://www.centreforpublicimpact.org/ case-study/electronic-health-records-system-uk/.

Batelle Memorial Institute. 2013. "The Impact of Genomics on the U.S. Economy.” Battelle Memorial Institute, Columbus, OH. https://web.ornl. gov/sci/techresources/Human_Genome/publica$\mathrm{t} / 2013$ BattelleReportImpact-of-Genomics-on-the-US-Economy.pdf.

Bray, Freddie, Jacques Ferlay, Isabelle Soerjomataram, Rebecca L. Siegel, Lindsey A. Torre, and Ahmedin Jemal. 2018. "Global Cancer Statistics 2018: GLOBOCAN Estimates of Incidence and Mortality Worldwide for 36 Cancers in 185 Countries." CA: A Cancer Journal for Clinicians 68 (6): 394-424. American Cancer Society. https://doi.org/10.3322/ caac.21492.

Center for Applied Policy and Integrity. 2015. "Strengthening Integrity and Fighting Corruption in Education: Armenia." Report prepared on behalf of the Open Society Foundations - Armenia, Center for Applied Policy and Integrity, Sofia. http://www.osf.am/wp-content/uploads/2016/o1/ Integrity-report_final_en_12.11.2015.pdf.

Coherent Market Insights. 2017. "Global Remote Patient Monitoring Devices Market.” Coherent Market Insights, Seattle. https://www.coherentmarketinsights.com/market-insight/ remote-patient-monitoring-market-215. Day, Sean, and Megan Zweig. 2019. "2018 Year End
Funding Report: Is Digital Health in a Bubble?" Rock Health, San Francisco. https://rockhealth.com/ reports/2018-year-end-funding-report-is-digital-health-in-a-bubble/.

Gerbert, Philipp, and Frank Rueß. 2018. "Quantum Computing — and How to Play.” Boston Consulting Group, Boston. https://www.bcg.com/en-us/ publications/2018/next-decade-quantum-computing-how-play.aspx.

Grand View Research. 2019. "Remote Patient Monitoring System Market Size, Share, and Trends Analysis Report." Grand View Research, San Francisco. https://www.grandviewresearch.com/ industry-analysis/ remote-patient-monitoring-devices-market.

Green, Harriet. 2018. "IBM Sees Quantum Computing Going Mainstream within Five Years.” Interview by Hadley Gamble. Capital Connection, CNBC, March 30. Video, 2:37. https://www.cnbc. com/2018/03/30/ibm-sees-quantum-computing-going-mainstream-within-five-years.html.

InfoDev. 2014. Building Competitive Green Industries: The Climate and Clean Technology Opportunity for Developing Countries. Washington, DC: World Bank. http://documents.worldbank.org/curated/ en/837201468165876160/pdf/9116o-REPLACEMENT-Building-Competitive-Green-Industries. pdf.

IP/IT Committee for Armenia. 2019. “Boost Armenia's Economic Development by Enhancing Innovation and by improving IP protection and enforcement framework."

Lapedus, Mark. 2018. "Foundry Challenges in 2018." Semiconductor Engineering, December 27. https:// semiengineering.com/foundry-challenges-in-2018/.

Markets and Markets. 2019. "Geotechnical Instrumentation and Monitoring Market." Markets and Markets, Pune. https://www.marketsandmarkets. com/Market-Reports/geotechnical-instrumentation-monitoring-market-221101423.html.

McCandless, David. 2017. "Codebases: Millons of Lines of Codes.” Information is Beautiful. https:// informationisbeautiful.net/visualizations/ million-lines-of-code/.

Medina, Leandro, and Friedrich Schneider. 2018. "Shadow Economies Around the World: What Did 
We Learn Over the Last 20 Years?” International Monetary Fund, Washington, D.C. https://www. imf.org/en/Publications/WP/Issues/2018/01/25/ Shadow-Economies-Around-the-World-What-Did-We-Learn-Over-the-Last-2o-Years-45583.

ON Semiconductor. 2019. "Implantable Medical Devices.” ON Semiconductor, Phoenix. https:// www.onsemi.com/PowerSolutions/content. do?id=16605\&parentApp=16602.

Pulidindi, Kiran, and Soumalya Chakraborty. 2019. “Seismic Reinforcement Materials Market.” Global Markets Insights, Selbyville, DE. https://www. gminsights.com/industry-analysis/ seismic-reinforcement-materials-market.

PWC (PricewaterhouseCoopers). 2017. "Semiconductor Industry: Strategies for Growth in the Internet of Things Era." PWC, London. https://www.strategyand.pwc.com/gx/en/insights/semiconductor-industry.html.

Rodrik, Dani. 2013. "Structural Change, Fundamentals, and Growth: An Overview.” Institute for Advanced Study, Princeton, NJ. http://j.mp/1HgaMHD.

—. 2014. "New Growth Strategies." Keynote presentation at the World Bank Group's headquarters, "New Growth Strategies: Delivering on their Promise?," Washington, D.C., October 14-15. https://www.theciip.org/sites/ciip/files/documents/ Keynote\%20-\%2OProf\%2ODani\%2ORodrik.pdf.

San Diego Regional EDC (Economic Development Corp). 2016. "Cracking the Code: The Economic Impact of San Diego's Genomics Industry.” BW Research Partnership, Carlsbad, CA. https://www. sandiegobusiness.org/sites/default/files/ Cracking\%2othe\%20Code\%20-\%20The\%20 Economic\%20Impact\%20of\%20San\%20 Diego's\%20Genomics\%2oIndustry_o.pdf.

Schmaltz, Remi. 2017. "What is Precision Agriculture." AgFunderNews, April 24. https://agfundernews.com/ what-is-precision-agriculture.html.

SIPRI (Stockholm International Peace Research Institute). 2019. "SIPRI Military Expenditure Database.” SIPRI, Solna. https://www.sipri.org/ databases/milex.

Sperling, Ed. 2017. “Is 7nm The Last Major Node?” Semiconductor Engineering, July 20. https://semiengineering.com/7nm-last-major-node/.

Stratistics Market Research Consulting Pvt Ltd. 2018. "Air Pollution Control Systems - Global Market Outlook (2017-2026)." Stratistics Market Research Consulting Pvt Ltd., Gaithersburg, MD. https://www.researchandmarkets.com/research/ sllx8c/global_air?w=5.

Sumant, Onkar, and Pallavi Jaiswal. 2016. "Remote Patient Monitoring Market by Condition.” Allied Market Research, Portland, OR. https://www. alliedmarketresearch.com/ remote-patient-monitoring-market.

Ugalmugale, Sumant, and Rupali Swain. 2019. "Digital Health Market.” Global Market Insights, Selbyville, DE. https://www.gminsights.com/industry-analysis/digital-health-market.

WHO (World Health Organization). 2014. "Noncommunicable Diseases Country Profiles 2014.” WHO, Geneva. https://www.who.int/nmh/publications/ ncd-profiles-2014/en/.

World Bank. 2013. "Armenia - BEEPS at a Glance 2013." World Bank, Washington, D.C. http://documents. worldbank.org/curated/en/699591468218377943/ Armenia-BEEPS-at-a-glance-2013.

- 2017. Armenia - Systematic Country Diagnostic: Sustainable, Inclusive Agriculture Sector Growth in Armenia: Lessons from Recent Experience of Growth and Contraction. Washington, D.C.: World Bank Group.

—. 2019. "Access to Finance - Technical Note.” World Bank, Washington, D.C. 


\section{Contact}

Ifeyinwa Uchenna Onugha

ionugha@worldbank.org 